\title{
IMPLEMENTATION OF THE SHEARING BOX APPROXIMATION IN ATHENA
}

\author{
JAMES M. STONE \\ Department of Astrophysical Sciences, Princeton University, Princeton, NJ 08544 \\ AND THOMAS A. GARDINER \\ Sandia National Laboratories, Albuquerque, NM 87185-1189 \\ Draft version May 29, 2018
}

\begin{abstract}
We describe the implementation of the shearing box approximation for the study of the dynamics of accretion disks in the Athena magnetohydrodynamics (MHD) code. Second-order Crank-Nicholson time differencing is used for the Coriolis and tidal gravity source terms that appear in the momentum equation for accuracy and stability. We show this approach conserves energy for epicyclic oscillations in hydrodynamic flows to round-off error. In the energy equation, the tidal gravity source terms are differenced as the gradient of an effective potential in a way which guarantees that total energy (including the gravitational potential energy) is also conserved to round-off error. We introduce an orbital advection algorithm for MHD based on constrained transport to preserve the divergence-free constraint on the magnetic field. This algorithm removes the orbital velocity from the time step constraint, and makes the truncation error more uniform in radial position. Modifications to the shearing box boundary conditions applied at the radial boundaries are necessary to conserve the total vertical magnetic flux. In principle similar corrections are also required to conserve mass, momentum and energy, however in practice we find the orbital advection method conserves these quantities to better than $0.03 \%$ over hundreds of orbits. The algorithms have been applied to studies of the nonlinear regime of the MRI in very wide (up to 32 scale heights) horizontal domains.
\end{abstract}

Subject headings: hydrodynamics, MHD, methods:numerical

\section{INTRODUCTION}

Numerical methods are an important tool for studying the nonlinear gas dynamics in accretion flows. For example, much of what we have learned about the saturation of the magnetorotational instability (MRI) has come from magnetohydrodynamic (MHD) simulations (Balbus \& Hawley 2003). A large fraction of such calculations adopt a local approximation (Hill 1878) termed the "shearing box", first introduced in studies of the MRI by Hawley et al. (1995, hereafter HGB). In this approximation, the equations of motion are written in a local, Cartesian reference frame co-rotating with the disk at some arbitrary radius $r_{0}$. The approximation is valid provided the linear extent of the domain under study is small compared to $r_{0}$. The shearing box approximation has limitations (Regev \& Umurhan 2008), in particular it cannot be used to calculate important global properties of the disk like the net mass accretion rate, radial density and temperature profiles, or the spectrum of emitted radiation. Nevertheless it has provided a useful laboratory for the study of important questions related to the local dynamics of accretion disks (Balbus 2003). For example, recent studies that use the shearing box include the effect of microscopic diffusivities on the saturation level of MHD turbulence driven by the MRI (Fromang et al. 2007; Lesur \& Longaretti 2007; Simon \& Hawley 2009), the saturation of the MRI in radiation pressure dominated disks (Hirose et al. 2008), and the effects of non-ideal MHD and dust on the properties of the MRI in protostellar disks (Turner \& Sano 2008; Ilgner \& Nelson 2008).
The shearing box approximation requires source terms be added to the momentum and energy equations, and special boundary conditions be used in the radial direction. Most of the simulations of the MRI presented to date have used operatorsplit methods like ZEUS (e.g. HGB; Stone et al. 1996), or simple finite-difference methods like the PENCIL code (e.g. Johansen et al. 2009). In both cases, adding the shearing box source terms is straightforward (HGB). More recently, higherorder Godunov methods that adopt the conservative form have begun to be applied to studies of accretion flows in the shearing box (Shen et al. 2006; Fromang \& Papaloizou 2007; Bodo et al. 2008; Piontek et al. 2009; Simon et al. 2009; Tilley et al. 2009; Gressel 2010).

In this paper, we provide a detailed description of the algorithmic extensions for the shearing box approximation in Athena, a recently developed higher-order Godunov code for astrophysical MHD. The basic MHD algorithms in Athena are documented in Gardiner \& Stone (2005a; 2008), and details of the implementation and tests of the methods are given in Stone et al. (2008, hereafter SGTHS) and Stone \& Gardiner (2009). Currently there are two versions of Athena, one implemented in $\mathrm{C}$ and the other in Fortran. An extension of the Fortran version for the shearing box has already been used for new studies of the MRI (Simon \& Hawley 2009; Simon et al. 2009). The numerical algorithms in the $\mathrm{C}$ version are slightly different. They were first introduced by Gardiner \& Stone (2005b), and used to study hydrodynamic shearing waves by Shen et al. 
(2006) and Balbus \& Hawley (2006). They have the advantage of preserving the energy integral in epicyclic motion to round-off, conserving the total (including gravitational potential) energy to round-off, and producing virtually no aliasing of trailing into leading waves. We emphasize, however, that since epicyclic motion is destabilized by a weak magnetic field (Balbus \& Hawley 1991), the accuracy of this approach can only be quantitatively demonstrated for hydrodynamic flows. Although similar methods were adopted and extended by Gressel \& Ziegler (2007), this paper provides the first comprehensive description of the algorithms in the $\mathrm{C}$ version of Athena. The $\mathrm{C}$ version is also being used for new studies of the MRI (Davis et al. 2010).

In addition to methods for the shearing box source terms, we also describe the implementation of an orbital advection algorithm for MHD in Athena. Orbital advection can greatly increase the efficiency of calculations in domains that span more than one scale height in the radial direction (so that the difference in the orbital speed across the domain is supersonic), since the time step constraint for stability does not depend on the magnitude of the local orbital velocity, but only on the amplitude of the fluctuations in the velocity around this value. Moreover, orbital advection can improve the accuracy of the integration by making the truncation error more uniform in radius (Johnson et al. 2008, hereafter JGG). Orbital advection was first introduced for hydrodynamic studies of disks by Masset (2000) in the FARGO code. More recently, JGG have described an extension of orbital advection methods to MHD for ZEUS-type codes. Johansen et al. (2009) have described the extension of the PENCIL code with orbital advection using Fourier transform methods. The method we have implemented in Athena is quite different from these previous approaches. In particular, we update the magnetic field using constrained transport (Evans \& Hawley 1988) to guarantee the divergence-free constraint is enforced to machine precision, using an effective electric field produced by the orbital motion. This greatly simplifies the method.

The organization of this paper is as follows. In the following section, we catalog the basic equations solved by Athena in the shearing box approximation. In $\S 3$ we describe our implementation of the source terms in these equations, including tests of our methods. In $\S 4$ we describe the shearing box boundary conditions for the conservative variables, including issues associated with parallelization of the algorithms with MPI. Finally, in $\S 5$ we describe our orbital advection method, along with tests. We present preliminary simulations of the MRI in very wide horizontal domains and summarize in $\S 6$.

\section{BASIC EQUATIONS}

The local shearing box approximation adopts a frame of reference located at radius $r_{0}$ corotating with the disk at orbital frequency $\Omega_{0}=\Omega\left(r_{0}\right)$. In this frame, the equations of MHD are written in a Cartesian coordinate system $(x, y, z)$ that has unit vectors $\hat{\mathbf{i}}, \hat{\mathbf{j}}$, and $\hat{\mathbf{k}}$ as

$$
\begin{aligned}
\frac{\partial \rho}{\partial t}+\nabla \cdot[\rho \mathbf{v}] & =0, \\
\frac{\partial \rho \mathbf{v}}{\partial t}+\nabla \cdot[\rho \mathbf{v v}+\mathrm{T}] & =\rho \Omega_{0}^{2}(2 q x \hat{\mathbf{i}}-z \hat{\mathbf{k}})-2 \Omega_{0} \hat{\mathbf{k}} \times \rho \mathbf{v}, \\
\frac{\partial E}{\partial t}+\nabla \cdot[E \mathbf{v}+\mathrm{T} \cdot \mathbf{v}] & =\Omega_{0}^{2} \rho \mathbf{v} \cdot(2 q x \hat{\mathbf{i}}-z \hat{\mathbf{k}}), \\
\frac{\partial \mathbf{B}}{\partial t}-\nabla \times(\mathbf{v} \times \mathbf{B}) & =0,
\end{aligned}
$$

where $T$ is the total stress tensor

$$
\mathrm{T}=\left(p+B^{2} / 2\right) \mathbf{I}-\mathbf{B B},
$$

$p$ is the gas pressure, $E$ is the sum of the internal, kinetic, and magnetic energy densities

$$
E=\frac{p}{\gamma-1}+\frac{1}{2} \rho v^{2}+\frac{B^{2}}{2},
$$

and $v^{2}=\mathbf{v} \cdot \mathbf{v}, B^{2}=\mathbf{B} \cdot \mathbf{B}$. The shear parameter $q$ is defined as

$$
q=-\frac{1}{2} \frac{d \ln \Omega^{2}}{d \ln r}
$$

so that for Keplerian flow $q=3 / 2$. The other symbols have their usual meaning. These equations are written in units such that the magnetic permeability $\mu=1$, and an equation of state appropriate to an ideal gas has been assumed in writing equation 6 that is $p=(\gamma-1) e$ (where $\gamma$ is the ratio of specific heats, and $e$ is the internal energy density). The techniques described in this paper are easily generalized to other equations of state.

The first two source terms on the RHS of equation 2 represent the radial and vertical components of the gravitational force in the rotating frame, while the third term represents the Coriolis force. In the energy equation 3 , the two source terms on the RHS represent the work done by the radial and vertical components of gravity in the rotating frame. Most of the challenges associated with the addition of the shearing box source terms to Godunov schemes like Athena are related to the tidal gravity and Coriolis terms which act in the orbital $(x-y)$ plane, therefore in the discussion below we describe the algorithms for the source terms in the horizontal and vertical directions separately.

The equations of motion in the shearing box admit a simple equilibrium solution representing uniform orbital motion,

$$
\mathbf{v}_{K}=-q \Omega_{0} x \hat{\mathbf{j}} .
$$

Note this velocity is time-independent, varies only in the $x$-direction, and only the $y$-component is non-zero. These properties are important for the orbital advection scheme described in $\S 5$.

\section{SHEARING BOX SOURCE TERMS}

One approach to implementing source terms in Godunov schemes like Athena is to operator split them from the flux divergence terms, resulting in a system of ODEs that can be integrated with any number of methods. For example, Simon et al. (2009) have successfully used this approach to study the saturation of the MRI with the Fortran version of Athena. However, in studies of decaying hydrodynamic turbulence in the shearing 
box (Shen et al. 2006), we found the kinetic energy in velocity fluctuations could increase at very late times, rather than decay monotonically to zero. We traced the source of this growth to an artificial amplification of epicyclic motions by the truncation error associated with the source terms in the momentum equations. As we show below, in hydrodynamics it is possible to develop a discretization that will instead conserve this energy to round-off error. While this approach may not provide significant improvement for MHD flows, since epicyclic motion is unstable with weak magnetic fields, we show below it is more accurate for hydrodynamics, and therefore we have adopted it as the default algorithm in both hydrodynamics and MHD. The methods for integrating the source terms described below were first introduced by Gardiner \& Stone (2005b), subsequently Gressel \& Ziegler (2007) have adopted and expanded upon this approach.

To describe our methods, it is useful to define the $x$ - and $y$-components of the momentum density fluctuations

$$
\begin{gathered}
m=\rho v_{x} \\
n=\rho\left(v_{y}+q \Omega_{0} x\right)
\end{gathered}
$$

To investigate the properties of epicyclic motion in a stress-free medium, we consider the case in which the velocity fluctuation is a function of time and rewrite the momentum equation 2 using these variables, giving

$$
\begin{gathered}
\frac{\partial m}{\partial t}=2 \Omega_{0} n \\
\frac{\partial n}{\partial t}=(q-2) \Omega_{0} m
\end{gathered}
$$

Multiplying equation 11 by $m$, equation 12 by $-2 n /(q-2)$, and adding gives

$$
\frac{\partial}{\partial t}\left(m^{2}+\frac{2}{2-q} n^{2}\right)=0
$$

Thus, there is a conserved energy which for Keplerian flow $(q=3 / 2)$ is $E_{\text {epi }}=\left[m^{2}+4 n^{2}\right] /(2 \rho)$.

We would like the numerical discretization of the momentum equation to conserve $E_{\text {epi }}$ exactly. Remarkably, nothing more complicated than Crank-Nicholson time differencing is required. Following the usual convention, we discretize time into $N$ non-uniform steps, and use a superscript to denote the time level of any quantity, with the timestep $\delta t$ defined as $\delta t=t^{n+1}-t^{n}$. We define time difference and averaging operators as

$$
\begin{gathered}
{[x]=x^{n+1}-x^{n}} \\
\bar{x}=\frac{1}{2}\left(x^{n+1}+x^{n}\right)
\end{gathered}
$$

respectively. Then, the Crank-Nicholson time difference formulae for equations 11 and 12 are

$$
\begin{gathered}
{[m]=2 \Omega_{0} \delta t \bar{n}} \\
{[n]=(q-2) \Omega_{0} \delta t \bar{m}}
\end{gathered}
$$

By multiplying the first of these equations by $\bar{m}$, the second by $-2 \bar{n} /(q-2)$, and adding gives, after some manipulation

$$
\left[m^{2}+\frac{2}{2-q} n^{2}\right]=0 .
$$

Thus, Crank-Nicholson discretization conserves the energy integral for epicyclic oscillations in a discrete sense. There is a simple physical interpretation of this result. The Coriolis force $\mathbf{F}_{c}=(-2 \Omega \times \mathbf{v})$, hence $\mathbf{v} \cdot \mathbf{F}_{c}=0$ and the Coriolis force can do no work. Without care, the discretized Coriolis force may not be orthogonal to the velocity (in a time averaged sense). This can lead to unphysical growth or decay of the energy in epicyclic motion. Our tests have revealed that a forward Euler discretization leads to a growing amplitude for epicyclic oscillations, while a backward Euler discretization leads to a decaying amplitude. Fortunately, the average of the two (Crank-Nicholson differencing), conserves the energy. Similarities can be drawn between integrating fluid motion due to the Coriolis force, and particle orbits in a central potential, e.g. forward Euler applied to the latter leads to outward spiraling rather than closed orbits.

\subsection{Source Terms in the Momentum Equation}

We now develop a finite-volume discretization of the momentum equation 2, using a Crank-Nicholson time discretization of the tidal gravity and Coriolis source terms. We consider only the $x$ - and $y$-components of this equation. Written in terms of the momentum density fluctuations, $m$ and $n$ respectively, these equations are

$$
\begin{gathered}
\frac{\partial m}{\partial t}+\nabla \cdot \mathbf{F}_{m}=2 \Omega_{0} n \\
\frac{\partial n}{\partial t}+\nabla \cdot \mathbf{F}_{n}=(q-2) \Omega_{0} m
\end{gathered}
$$

where $\mathbf{F}_{m}$ and $\mathbf{F}_{n}$ are vectors whose components are the fluxes of the momentum density fluctuations in each direction. These are related to the vector of fluxes of the corresponding components of the momentum density, $\mathbf{F}_{\rho v_{x}}$ and $\mathbf{F}_{\rho v_{y}}$ respectively, via

$$
\begin{gathered}
\mathbf{F}_{m}=\mathbf{F}_{\rho v_{x}} \\
\mathbf{F}_{n}=\mathbf{F}_{\rho v_{y}}+q \Omega_{0} x \mathbf{F}_{\rho}
\end{gathered}
$$

We now adopt a finite-volume discretization of equations 19 and 20 (see $\$ 3$ in SGTHS for a discussion of this approach). We use indices $(i, j, k)$ to denote spatial locations on a discrete grid with cell centered locations $\left(x_{i}, y_{j}, z_{k}\right)$. Half integer indices are used to denote the appropriate cell interfaces. The dependent variables stored on this mesh $m_{i, j, k}^{n}$ and $n_{i, j, k}^{n}$ are understood to be volume averaged values in the sense of equation 12 in SGTHS. Integrating over a timestep $\delta t=t^{n+1}-t^{n}$ and over the volume of a cell, equations 19 and 20 become

$$
\begin{gathered}
\left.\frac{\left(m_{i, j, k}^{n+1}-m_{i, j, k}^{n}\right)}{\delta t}+\overline{\left(\nabla \cdot \mathbf{F}_{m}\right.}\right)_{i, j, k}=2 \Omega_{0} \frac{\left(n_{i, j, k}^{n}+n_{i, j, k}^{n+1}\right)}{2} \\
\frac{\left(n_{i, j, k}^{n+1}-n_{i, j, k}^{n}\right)}{\delta t}+{\overline{\left(\nabla \cdot \mathbf{F}_{n}\right.}}_{i, j, k}=(q-2) \Omega_{0} \frac{\left(m_{i, j, k}^{n}+m_{i, j, k}^{n+1}\right)}{2}
\end{gathered}
$$

In a finite volume approach, the time- and volume-average of the flux divergence (the second term in both equations) would be rewritten, using the divergence theorem, as the difference of the time- and area-averaged fluxes at each of the cell faces, e.g. equations 11-15 in SGTHS. In a Godunov scheme, these fluxes are computed with a Riemann solver. For brevity, we 
have suppressed expanding the flux divergence terms into these differences, with the understanding that our notation is meant to represent these terms.

Solving equation 24 for $\left(n_{i, j, k}^{n}+n_{i, j, k}^{n+1}\right)$ and substituting into equation 23 gives, after some manipulation

$$
\begin{aligned}
m_{i, j, k}^{n+1} & =m_{i, j, k}^{n}-\delta t{\overline{\left(\nabla \cdot \mathbf{F}_{m}\right)_{i, j, k}}}+\left(\frac{4 \Omega_{0} \delta t}{2+(2-q)\left(\Omega_{0} \delta t\right)^{2}}\right)\left(\left\{n_{i, j, k}^{n}-\frac{\delta t}{2} \overline{\left(\nabla \cdot \mathbf{F}_{n}\right)_{i, j, k}}\right\}\right. \\
& \left.+\frac{(q-2)}{2} \Omega_{0} \delta t\left\{m_{i, j, k}^{n}-\frac{\delta t}{2} \overline{\left(\nabla \cdot \mathbf{F}_{m}\right)_{i, j, k}}\right\}\right)
\end{aligned}
$$

Similarly, solving equation 23 for $\left(m_{i, j, k}^{n}+m_{i, j, k}^{n+1}\right)$ and substituting into equation 24 gives

$$
\begin{aligned}
n_{i, j, k}^{n+1} & =n_{i, j, k}^{n}-\delta t{\overline{\left(\nabla \cdot \mathbf{F}_{n}\right)_{i, j, k}}}+\left(\frac{2(q-2) \Omega_{0} \delta t}{2+(2-q)\left(\Omega_{0} \delta t\right)^{2}}\right)\left(\left\{m_{i, j, k}^{n}-\frac{\delta t}{2} \overline{\left(\nabla \cdot \mathbf{F}_{m}\right)_{i, j, k}}\right\}\right. \\
& \left.+\Omega_{0} \delta t\left\{n_{i, j, k}^{n}-\frac{\delta t}{2} \overline{\left(\nabla \cdot \mathbf{F}_{n}\right)_{i, j, k}}\right\}\right)
\end{aligned}
$$

In order to convert these update equations for $m_{i, j, k}$ and $n_{i, j, k}$ respectively, into update equations for $\left(\rho v_{x}\right)_{i, j, k}$ and $\left(\rho v_{y}\right)_{i, j, k}$ (the discrete form of the conserved quantities actually updated in the code), relationships between the discrete fluxes of these quantities are required. For any cell $(i, j, k)$ with zone-center $x$-position $x_{i}$, the relationships between the components of the volume averaged momentum density fluctuations and the momentum density are

$$
\begin{gathered}
m_{i j k}=\left(\rho v_{x}\right)_{i j k} \\
n_{i j k}=\left(\rho v_{y}\right)_{i j k}+q \Omega_{0} \rho_{i j k} x_{i}
\end{gathered}
$$

From equations 21 and 22 the divergence of the fluxes of these quantities are related via

$$
\begin{gathered}
\nabla \cdot \mathbf{F}_{m}=\nabla \cdot \mathbf{F}_{\rho v_{x}} \\
\nabla \cdot \mathbf{F}_{n}=\nabla \cdot \mathbf{F}_{\rho v_{y}}+q \Omega_{0} \nabla \cdot\left(x \mathbf{F}_{\rho}\right)
\end{gathered}
$$

which can be written in a finite volume discretization as

$$
\begin{aligned}
& {\overline{\left(\nabla \cdot \mathbf{F}_{m}\right)_{i, j, k}}}_{\overline{\left(\nabla \cdot \mathbf{F}_{\rho v_{x}}\right)_{i, j, k}}} \\
& {\overline{\left(\nabla \cdot \mathbf{F}_{n}\right)_{i, j, k}}}_{\overline{\left(\nabla \cdot \mathbf{F}_{\rho v_{y}}\right)_{i, j, k}}}+q \Omega_{0} \bar{x}_{i}{\overline{\left(\nabla \cdot \mathbf{F}_{\rho}\right)_{i, j, k}}} \\
& +(q / 2) \Omega_{0} x_{i}\left(\left(F_{\rho}\right)_{x, i-1 / 2}+\left(F_{\rho}\right)_{x, i+1 / 2}\right)
\end{aligned}
$$

Finally, inserting equations 28 and 32 into 26 gives

$$
\begin{aligned}
\left(\rho v_{y}\right)_{i, j, k}^{n+1} & =\left(\rho v_{y}\right)_{i, j, k}^{n}-\delta t \overline{\left(\nabla \cdot \mathbf{F}_{\left(\rho v_{y}\right)}\right)} \\
& -\frac{q}{2} \Omega_{0} \delta t x_{i}\left(\left(F_{\rho}\right)_{x, i-1 / 2}+\left(F_{\rho}\right)_{x, i+1 / 2}\right) \\
& +\left(\frac{2(q-2) \Omega_{0} \delta t}{2+(2-q)\left(\Omega_{0} \delta t\right)^{2}}\right)\left(\left\{m_{i, j, k}^{n}-\frac{\delta t}{2} \overline{\left(\nabla \cdot \mathbf{F}_{m}\right)_{i, j, k}}\right\}\right. \\
& \left.+\Omega_{0} \delta t\left\{n_{i, j, k}^{n}-\frac{\delta t}{2} \overline{\left(\nabla \cdot \mathbf{F}_{n}\right)_{i, j, k}}\right\}\right)
\end{aligned}
$$

Since the $m_{i, j, k}$ and $\left(\rho v_{x}\right)_{i, j, k}$ are identical, the update relation for the latter is given by equation 25.
Equations 25 and 33 above represent the desired finite volume discretization of the momentum equation, where the divergence of the fluxes of the momentum fluctuations that appear in these equations are given in terms of the divergence of the fluxes of the conserved quantities actually returned by the Riemann solver by equations 31 and 32. As we will show through the tests described in $\$ 3.4$, the Crank-Nicholson time differencing of the shearing box source terms used in these equations conserves the energy integral associated with epicyclic motion exactly.

To implement these difference equations in a computer code additional algorithmic steps are required. For example, the reconstruction of the left- and right-interface states in the $x$-direction (see $\$ 4.2$ of SGTHS) which is needed to compute the time-and area averaged fluxes of the conserved variables at the $x$-interfaces using a Riemann solver requires the addition of shearing box source terms. In particular, before the left- and right-states in the primitive variables at $x$-interfaces are converted back to the conserved variables $\left(\mathbf{q}_{L, i-1 / 2}\right.$ and $\mathbf{q}_{R, i-1 / 2}$ in the notation of SGTHS) at the end of the first-, second-, or thirdorder reconstruction algorithms describe in $\$ 4.2$ of SGTHS, the shearing box source terms for the velocity components $v_{x}$ and $v_{y}$ must be added for $\delta t / 2$. We have found that simple forward Euler time-differencing is adequate for this step.

Similarly, the directionally unsplit corner transport upwind (CTU) integrator used in Athena (see §5.1 and §6.1 in SGTHS) uses transverse flux gradients to correct the interface states in multidimensions. For the flux gradients in the $x$-direction (added to the $y$-interface states in 2D, and the $y$ - and $z$-interface states in 3D), the appropriate shearing box source terms must be added to $x$ - and $y$-components of the momentum for $\delta t / 2$. Again, we have found that simple forward Euler differencing is adequate for this step.

Finally, in order to compute the cell-centered reference electric field at the half time step $\mathcal{E}_{i, j, k}^{r, n+1 / 2}$ needed for the CT algorithm (computed in step 5 of the 2D CTU algorithm, or step 6 in the 3D CTU algorithm), the shearing box source terms with forward Euler discretization must be added in the calculation of the velocity components at the half time step.

In summary, our algorithm for the momentum equation update in the shearing box approximation as implemented in Athena consists of the following modifications to the CTU+CT algorithm described in SGTHS:

1. Add shearing box source terms to the left- and rightstates for the velocity at $x$-interfaces only during reconstruction step, before converting the reconstructed primitive variables to conserved variables.

2. Add shearing box source terms to the $x$ - and $y$-components of the momentum when a transverse flux gradient in $x$-direction is applied to $y$ - and $z$-interface states as part of CTU integrator.

3. Add shearing box source terms to velocity when computing the cell-centered reference electric field at the half time step needed by the CT algorithm (see step 5 
in $\S 4.2$ of Gardiner \& Stone 2008).

4. Use equations 25 and 33 to evolve the momentum in the final conservative update, where the divergence of the fluxes of the momentum fluctuations come from equations 31 and 32 .

As we show with tests, the main advantage of this algorithm is that it conserves the energy integral for epicyclic motion exactly.

\subsection{Source Terms in the Energy Equation}

Next we consider the finite-volume discretization of the energy equation 3 including the shearing box source terms. The key to this discretization is to define an effective potential for the shearing box

$$
\Phi_{S B}=-q \Omega_{0}^{2} x^{2}+\frac{1}{2} \Omega_{0}^{2} z^{2}
$$

so that the source terms in the energy equation 3 can be written as

$$
\frac{\partial E}{\partial t}+\nabla \cdot[E \mathbf{v}+\mathbf{T} \cdot \mathbf{v}]=\rho \mathbf{v} \cdot \nabla \Phi_{S B}
$$

When written in this form, it is clear that the source term represents the rate of change in the gravitational potential energy per unit volume.

We seek a discretization of this term such that when integrated over volume, we recover the rate of change of energy due to the work done at the boundaries. This suggests that for each computational cell, we discretize the source term by the difference of the work done at the edges of the cell. Ignoring for the moment the vertical component of gravity, so that the effective potential depends only on $x$, the appropriate finite-volume discretization of equation 35 is

$$
\begin{aligned}
E_{i, j, k}^{n+1} & \left.=E_{i, j, k}^{n}-\delta t \overline{\left(\nabla \cdot \mathbf{F}_{E}\right.}\right)_{i, j, k} \\
& -\frac{\delta t}{2}\left(\left(F_{\rho}\right)_{x, i+1 / 2, j, k} \frac{\Phi_{S B, i+1 / 2, j, k}-\Phi_{S B, i, j, k}}{\delta x / 2}\right. \\
& \left.+\left(F_{\rho}\right)_{x, i-1 / 2, j, k} \frac{\Phi_{S B, i, j, k}-\Phi_{S B, i-1 / 2, j, k}}{\delta x / 2}\right)
\end{aligned}
$$

where $\left(F_{\rho}\right)_{x, i+1 / 2, j, k}$ are the mass fluxes in the $x$-direction returned by the Riemann solver at the $x$-interfaces. It is straightforward to confirm that when integrated over volume, the source term reduces to the net mass flux times the difference in the gravitational potential across the domain. Thus, the only route via which the total energy in the domain can change is through work done at the boundaries. Within the domain energy can be exchanged between its kinetic, magnetic, and thermal forms, but it cannot be lost as truncation error. Even though we evolve an energy variable that does not contain the gravitational potential energy, we use a discretization that conserves to machine precision the total energy, that is $E+\rho \Phi_{S B}$.

In addition to the use of the update equation 36 , one other algorithmic step is required to include the shearing box source terms in the energy equation. This step is to include source terms to the transverse flux gradient corrections to the left- and right-interface states for $E$ in the multidimensional CTU algorithm. To be more precise, when the flux gradients in the $x$-direction are added to the $y$-interface states of $E$ in 2D, and the $y$ - and $z$-interface states of $E$ in 3D, the appropriate shearing box source terms must included for $\delta t / 2$. Again, we have found that simple forward Euler differencing is adequate for this step. Note that since the reconstruction step uses the primitive $(p)$ rather than conserved $(E)$ variables, no additional source terms are required for the evolution of the energy equation in the reconstruction step.

Thus, the extensions to the algorithms for energy equation update in the shearing box approximation consists of the following modifications:

1. Add shearing box source terms to $E$ when a transverse flux gradient in $x$-direction is applied to $y$ - and $z$-interface states of $E$ as part of the CTU integrator.

2. Use equation 36 to evolve $E$ in the final conservative update.

As shown in Gardiner \& Stone (2005b) in studies of the MRI in the shearing box with Athena, with this algorithm the rate of change of $E$ is equal to the work done at the boundaries to machine precision.

\subsection{Including Vertical Gravity}

To simplify the discussion in the previous subsections, we considered only the two components of the momentum equation in the orbital $(x-y)$ plane, and the source term associated with the vertical component of gravity in the energy equation was ignored. We have shown that care is needed in the discretization of the source terms in these equations in order to correctly capture the dynamics of epicyclic motion. However, for the discretization of the gravitational acceleration term in the vertical $(z-)$ component of the momentum equation, and the work term associated with the vertical component of gravity in the energy equation, we have found less complicated methods are adequate.

In Athena, the vertical gravity terms in both the vertical component of the momentum and the energy equation are added by differencing the effective potential equation 34. For the vertical component of the momentum equation, several algorithmic steps are required:

1. At the end of the reconstruction step, vertical acceleration for $\delta t / 2$ is added to the left- and right-states of $v_{z}$ at $z$-interfaces using the vertical gradient of the potential.

2. When the transverse flux corrections in the $z$-direction are applied to the $x$ - and $y$-interface states as part of the CTU unsplit integrator, vertical acceleration for $\delta t / 2$ is added to $\rho v_{z}$ using the gradient of the potential.

3. When computing the cell-centered reference electric field at the half time step needed by the CT algorithm (see step 5 in $\S 4.2$ of Gardiner \& Stone 2008), vertical acceleration for $\delta t / 2$ is added to $v_{z}$ using the gradient of the potential. 
4. In the final conservative update, the vertical force for $\delta t$ is added to $\rho v_{z}$ using the gradient of the potential and the density evaluated at the half time step, $\left(\rho_{i, j, k}^{n}+\rho_{i, j, k}^{n+1}\right) / 2$.

For the energy equation, the final conservative update equation 36 must be extended to include a term $S_{E, z}$, where

$$
\begin{aligned}
S_{E, z} & =-\frac{\delta t}{2}\left(\left(F_{\rho}\right)_{z, i, j, k+1 / 2} \frac{\Phi_{S B, i, j, k+1 / 2}-\Phi_{S B, i, j, k}}{\delta z / 2}\right. \\
& \left.+\left(F_{\rho}\right)_{z, i, j, k-1 / 2} \frac{\Phi_{S B, i, j, k}-\Phi_{S B, i, j, k-1 / 2}}{\delta z / 2}\right)
\end{aligned}
$$

This leads to the following algorithmic steps:

1. Add shearing box source terms to $E$ when a transverse flux gradient in $z$-direction is applied to $x$ - and $y$-interface states of $E$ as part of the CTU integrator.

2. Use equation 36 , including the term given by equation 37 on the RHS, to evolve $E$ in the final conservative update.

Inclusion of the source terms for vertical gravity, along with appropriate boundary conditions in the vertical direction, allow studies of the MRI in stratified disks (e.g. Stone et al. 1996) to be continued using Athena (Davis et al. 2010).

\subsection{Tests of the Source Terms}

There are a variety of axisymmetric $(\partial / \partial y \equiv 0)$ solutions to the MHD equations in the shearing box that serve as useful tests of the implementation of the source terms. Non-axisymmetric solutions required a more sophisticated treatment of the boundary conditions, as described in the next section. All of the tests in this section use a periodic domain in the $(x-z)$ plane which spans $-L_{x} / 2 \leq x \leq L_{x} / 2$ and $-L_{z} / 2 \leq z \leq L_{z} / 2$. The orbital frequency $\Omega_{0}=10^{-3}$, the shear parameter $q=3 / 2$, and an isothermal equation of state is used with sound speed $C_{s}=10^{-3}$. Unless otherwise stated, the initial conditions consist of a uniform density medium with $\rho_{0}=1$, pressure $p=\rho_{0} C_{s}^{2}$, and orbital velocity $v_{y}(x, 0)=-q \Omega_{0} x$. Third-order reconstruction, the HLLC (for hydrodynamics) or HLLD (for MHD) Riemann solver, and the CTU+CT unsplit integrator are used in all the tests.

A good first test is the evolution of epicyclic oscillations. We choose the initial radial velocity $v_{x}=0.1 C_{s}$, where $C_{s}=10^{-3}$, in domains of size $L_{x}=L_{z}=1,10$, and 50, and evolve the flow for thousands of orbits using a grid of $64^{2}$. We vary the size of the domain while keeping the resolution constant in order to study the effect of very large timesteps on the accuracy of the integration algorithm. Figure 1 shows the oscillations in $v_{x}$ over the first 20 orbits in each run. In each case, we observe epicyclic motion with constant amplitude: the energy integral in epicyclic motion is conserved exactly. Note there is a small dispersion error in the largest domain. However, in this case the time step is so large that there are only 14 timesteps per orbit. Thus, the small dispersion error $(\lesssim 2 \%)$ observed in this case (due to the Crank-Nicholson differencing) is to be expected. Increasing the resolution, and therefore decreasing the timestep, makes this error converge at second order. The key result is that even with large timesteps, the amplitude of oscillations is constant. However, we emphasize that this result is relevant only for hydrodynamics, since with weak magnetic fields epicyclic motion is unstable.

A more quantitative test is provided by the propagation of nonlinear axisymmetric density waves in the shearing box (Fromang \& Papaloizou 2007). The profile of such waves is given by the solution to two ordinary differential equations; for this test we use a numerical solution (kindly provided by S. Fromang) on a grid of 40 points in a domain with $L_{x}=9.593$ with a wave with amplitude $\rho_{\max } / \rho_{0}=1.05$. Figure 2 shows the error in the density, defined as $\delta \rho(x, t)=(\rho(x, t)-\rho(x, 0)) / \rho(x, 0)$ after $t=10,50$, and 100 wave periods $T$, where $T=5254.31$. Note the error is smooth (there are no oscillations indicative of dispersion error), and never larger than $1.5 \%$. We also plot the time evolution of the error in the wave amplitude, defined as $\left(\epsilon-\epsilon_{0}\right) / \epsilon_{0}$, where $\epsilon=\max (\rho)-\rho_{0}$. After 100 wave periods, we find the amplitude has decreased by only $3 \%$, which is a measure of the low numerical diffusion rate in Athena. These results compare very favorably to the test results presented in Fromang \& Papaloizou (2007), which were computed at a much higher resolution.

Finally, another useful test is the nonlinear evolution of axisymmetric modes of the MRI with no net flux. We use a domain of size $L_{x}=L_{z}=1$, and an initially vertical field with $\mathbf{B}=\left(0,0,\left(2 p_{0} / \beta\right)^{1 / 2} \sin 2 \pi x / L x\right)$, where $\beta=4000$. This test is identical to run S1c in Hawley \& Balbus (1992). In figure 3, we show images of the azimuthal velocity fluctuations $v_{y}+q \Omega_{0} x$ at various times in the evolution. The growth and saturation of the MRI is evident, with transition to two-dimensional MHD turbulence at late times. In two dimensions, turbulence cannot be sustained by the MRI, and the magnetic energy eventually decays. The rate of decay depends on numerical diffusion, which in turn depends on the grid resolution and numerical algorithm. Previously, we have used the rate of decay of the poloidal magnetic energy after saturation to compare the numerical dissipation rates of ZEUS and Athena (Stone \& Gardiner 2005; Stone 2009), to show that Athena gives lower decay rates at the same grid resolution. Figure 4 compares the time evolution of the energy associated with the radial component of the magnetic field $B_{x}^{2} / 2$ for various resolutions and both second- and third-order reconstruction. At every resolution the third-order reconstruction is measurably more accurate, in the sense that the rate of decay of the magnetic energy is decreased. Perhaps the best indicator of the accuracy of the methods is the evolution at very early times, while the amplitude of the MRI is very small. The short period of decay (due to the expansion of regions with the highest magnetic pressure), followed by exponential growth over 10 orders of magnitude in energy from the initial (very small) amplitude indicates Athena has a very good dispersion relation for slow MHD waves (which was also shown in the Stone et al. 2008 linear wave convergence tests).

\section{SHEARING BOX BOUNDARY CONDITIONS}

As first discussed in HGB, non-axisymmetric solutions in the shearing box require special boundary conditions that offset the 
solutions by the distance the radial edges of the domain have been displaced by the background shear. Mathematically, the shearing box boundary conditions can be expressed as (HGB; Gressel \& Ziegler 2007):

$$
f(x, y, z) \longmapsto f\left(x \pm L_{x}, y \mp w t, z\right)
$$

where $f=\left(\rho, \rho v_{x}, \rho v_{z}, \mathbf{B}\right)$, and

$$
\begin{gathered}
\rho v_{y}(x, y, z) \longmapsto \rho v_{y}\left(x \pm L_{x}, y \mp w t, z\right) \mp \rho w \\
E(x, y, z) \longmapsto E\left(x \pm L_{x}, y \mp w t, z\right) \mp \rho v_{y} w+\rho w^{2} / 2
\end{gathered}
$$

where $w=q \Omega_{0} L_{x}$ is the difference in the orbital velocity across the domain. Although a variety of authors have already described the implementation of the shearing box boundary conditions in various codes (e.g. HGB; Gressel \& Ziegler 2007), the details of the implementation can be important, thus we describe our methods below.

In Athena, we implement the shearing box boundary conditions by first applying periodic boundary conditions in the radial direction, (with the appropriate shift in the $y$-momentum and energy given by equations 39 and 401, and then use a conservative remap of all quantities in the ghost cells in the $y$-direction by a displacement $w t$, the distance the boundaries have sheared in time $t$. This distance in general is not an integer number of cells. To remap the solution a fractional portion of a cell we use the same higher order reconstruction functions as used in the integrator to compute the amount of each conserved variable to assign to the remapped cells. For the magnetic field, we remap each component in the ghost zones directly, rather than evolving the field in the ghost zones using a remap of the emfs (e.g. Gressel \& Ziegler 2007). This procedure does not guarantee the divergence free constraint is maintained in the ghost zones. However, since these cells are never used for anything more than interpolation, we have not found this to be a problem.

As pointed out by Gressel \& Ziegler (2007), the shearing box boundary conditions can destroy conservation, because the integral of the fluxes of the conserved quantities over the two radial faces may not be identical due to the remap. In particular, if the integral in the $y$-direction of the azimuthal component of the EMF at each radial face is not equal, then the net vertical flux in the domain is not conserved. Since the dynamics of the MRI is sensitive to the amplitude of the net vertical flux, this can represent a serious problem. To circumvent this issue, we remap the azimuthal component of the emf at each radial face, and use the arithmetic average of $\mathcal{E}_{y}$ computed for each grid cell at each radial face, and the remapped value of $\mathcal{E}_{y}$ from the corresponding grid cell on the opposite face, to update the magnetic field in the cells next to the boundary. Since the remap conserves the integral of $\mathcal{E}_{y}$ in the $y$-direction, the integral of the averaged $\mathcal{E}_{y}$ used to update the field on each boundary will be identical, and so this procedure conserves the net flux in the vertical direction to machine precision. In principle, this same procedure could be used for the fluxes of all the conserved quantities, to restore conservation in the domain. In particular, it can also be used to remap $\mathcal{E}_{z}$, so that the volume averaged azimuthal flux of the magnetic field is conserved to machine precision. In practice, we find that with the orbital advection algorithm described in $\S 5$, the hydrodynamic variables are conserved accurately even without this extra step, so that we generally only correct $\mathcal{E}_{y}$ in this fashion.

In the same way that the net vertical flux in the domain is not conserved without a special treatment of the remap of $\mathcal{E}_{y}$, the net toroidal flux is not conserved without a special treatment of the remap of $\mathcal{E}_{z}$. We have run a variety of simulations of the MRI in three dimensions, and find the net toroidal flux introduced in this way is very small: the magnetic energy associated with this flux shows random fluctuations with peak amplitude of around $10^{-9}$ of the thermal pressure for a zero-net-vertical flux simulation with an initial $\beta=4000$. However, we have found that matching the order of the reconstruction used in the ghost cells with the order used in the integrator, that is using third-order reconstruction in the ghost cells when third-order reconstruction is used throughout the rest of the domain, is important to keep this anomalous flux small. Using second-order reconstruction in the ghost zones produced a mean toroidal flux that is still very small, but 10 times larger than when third-order reconstruction is used in the ghost zones.

Efficient use of a large number $\left(\gtrsim 10^{3}\right)$ of processors on modern parallel computers requires domain decomposition of the grid in all three coordinate directions. However, parallelization of the shearing box boundary conditions can require complex book-keeping if domain decomposition in the azimuthal direction is allowed, because the data in the ghost zones for each processor on the radial boundaries can require communication with up to 3 processors on the opposite boundary, depending on the configuration of the domains as they shear. To simplify the implementation, we first apply periodic boundary conditions (which are easily parallelized) to pass ghost cells between processors in the radial direction, followed by a remap in the azimuthal direction. The latter is easily parallelized using a cyclic shift operation across those processors that store neighboring grid patches in the azimuthal direction. The cyclic shift is also easily parallelized, and always occurs between the same set of processors along the boundary. The disadvantage of this method is that it adds extra MPI calls, however this is more than offset by the reduction in complexity of the code, and the fact that the resulting communication pattern is more regular and therefore in most circumstances likely to be more efficient.

\section{ORBITAL ADVECTION}

Orbital advection methods (Masset 2000; JGG; Johansen et al. 2009) exploit the fact that the background orbital motion $\mathbf{v}_{K}$ is time-independent and varies only in radius (the $x$-coordinate in the shearing box). Thus, by decomposing the velocity into two parts

$$
\mathbf{v}=\mathbf{v}_{K}+\mathbf{v}^{\prime}
$$

where the components of the velocity fluctuation vector $\mathbf{v}^{\prime}$ are

$$
\left[\begin{array}{c}
v_{x}^{\prime} \\
v_{y}^{\prime} \\
v_{z}^{\prime}
\end{array}\right]=\left[\begin{array}{c}
v_{x} \\
v_{y}+q \Omega_{0} x \\
v_{z}
\end{array}\right]
$$


the equations of MHD in the shearing box (equations 1 through (4) can be rewritten as

$$
\begin{aligned}
\frac{\partial \rho}{\partial t}+v_{K} \frac{\partial \rho}{\partial y}+\nabla \cdot\left[\rho \mathbf{v}^{\prime}\right] & =0 \\
\frac{\partial \rho \mathbf{v}^{\prime}}{\partial t}+v_{K} \frac{\partial \rho \mathbf{v}^{\prime}}{\partial y}+\nabla \cdot\left[\rho \mathbf{v}^{\prime} \mathbf{v}^{\prime}+\mathrm{T}\right] & =2 \Omega_{0}\left(\rho v_{y}^{\prime}\right) \hat{\mathbf{i}}+(q-2) \Omega_{0}\left(\rho v_{x}^{\prime}\right) \hat{\mathbf{j}}-\rho \Omega \\
\frac{\partial E^{\prime}}{\partial t}+v_{K} \frac{\partial E^{\prime}}{\partial y}+\nabla \cdot\left[E^{\prime} \mathbf{v}^{\prime}+\mathrm{T} \cdot \mathbf{v}^{\prime}\right] & =\rho \mathbf{v}^{\prime} \cdot \nabla \Phi_{\mathrm{SB}}^{\prime}+\left(B_{x} B_{y}-\rho v_{x}^{\prime} v_{y}^{\prime}\right) \frac{\partial v_{K}}{\partial x}(4) \\
\frac{\partial \mathbf{B}}{\partial t}-\nabla \times\left(\mathbf{v}_{K} \times \mathbf{B}\right) & -\nabla \times\left(\mathbf{v}^{\prime} \times \mathbf{B}\right)=0
\end{aligned}
$$

where the total energy $E^{\prime}$ contains the kinetic energy in the velocity fluctuations (but not the background shear flow)

$$
E^{\prime}=\frac{p}{\gamma-1}+\frac{1}{2} \rho\left(\mathbf{v}^{\prime} \cdot \mathbf{v}^{\prime}\right)+\frac{B^{2}}{2},
$$

and the effective potential for the shearing box in the orbiting frame contains only the vertical gravity

$$
\Phi_{S B}^{\prime}=\frac{1}{2} \Omega_{0}^{2} z^{2}
$$

Note the second term on the LHS in each of equations 43 through 46 is a linear advection term with characteristic speed $v_{K}$. This suggests a numerical algorithm based on splitting these equations into two systems, the first containing only the linear advection operators

$$
\begin{aligned}
\frac{\partial \rho}{\partial t}+v_{K} \frac{\partial \rho}{\partial y} & =0, \\
\frac{\partial \rho \mathbf{v}^{\prime}}{\partial t}+v_{K} \frac{\partial \rho \mathbf{v}^{\prime}}{\partial y} & =0 \\
\frac{\partial E^{\prime}}{\partial t}+v_{K} \frac{\partial E^{\prime}}{\partial y} & =\left(B_{x} B_{y}-\rho v_{x}^{\prime} v_{y}^{\prime}\right) \frac{\partial v_{K}}{\partial x} \\
\frac{\partial \mathbf{B}}{\partial t}-\nabla \times\left(\mathbf{v}_{K} \times \mathbf{B}\right) & =0 .
\end{aligned}
$$

Note the extra terms on the RHS in the energy equation. These terms cannot be written as flux gradients, and must be treated as source terms. We discuss them in more detail below.

The remaining system of equations are the usual equations of MHD, written in terms of the velocity fluctuations $\mathbf{v}^{\prime}$ rather than the velocity $\mathbf{v}$, that is

$$
\begin{aligned}
\frac{\partial \rho}{\partial t}+\nabla \cdot\left[\rho \mathbf{v}^{\prime}\right] & =0 \\
\frac{\partial \rho \mathbf{v}^{\prime}}{\partial t}+\nabla \cdot\left[\rho \mathbf{v}^{\prime} \mathbf{v}^{\prime}+\mathrm{T}\right] & \left.=2 \Omega_{0}\left(\rho v_{y}^{\prime}\right) \hat{\mathbf{i}}+(q-2) \Omega_{0}\left(\rho v_{x}^{\prime}\right) \hat{\mathbf{j}}-\rho \Omega_{5}^{2} 5 \hat{\mathbf{k}}\right) \\
\frac{\partial E^{\prime}}{\partial t}+\nabla \cdot\left[E^{\prime} \mathbf{v}^{\prime}+\mathrm{T} \cdot \mathbf{v}^{\prime}\right] & =\rho \mathbf{v}^{\prime} \cdot \nabla \Phi_{\mathrm{SB}}^{\prime} \\
\frac{\partial \mathbf{B}}{\partial t}-\nabla \times\left(\mathbf{v}^{\prime} \times \mathbf{B}\right) & =0
\end{aligned}
$$

Note that since these equations written using $\rho v_{y}^{\prime}$ and $E^{\prime}$ as the conserved variables, instead of $\rho v_{y}$ and $E$, the shearing box source terms are modified compared to the original system equations 1 through 4 .

Developing numerical algorithms to solve these two systems of equations is straightforward. In particular, since the second system equations 53 through 56 are just the usual equations of MHD, but written in terms of a vector of conserved variables $\mathbf{q}=\left(\rho, \rho \mathbf{v}^{\prime}, E^{\prime}, \mathbf{B}\right)$, the standard CTU+CT algorithm in Athena (430an be used to integrate these equations, with the appropriate Crank-Nicholson time differencing of the shearing box source 2 . ent of the effective gravitational potential in the energy equation. These source terms are in fact simpler than in the original system, and the techniques described in $\$ 3$ apply directly. Since the variables being updated are the momentum fluctua(46ions themselves, the source terms added to the reconstruction step and transverse flux gradient corrections (see §3.2) are modified appropriately. Moreover, since the Riemann solver now returns the fluxes of the momentum fluctuations directly, the conversions in equations 31 and 32 are no longer necessary. Note that the CFL stability condition for this system is based only on the amplitude of the velocity fluctuations, that is

$$
\delta t=C_{\circ} \min \left(\frac{\delta x}{\left(\left|v_{x}^{\prime}\right|+C_{f x}\right)_{i, j, k}}, \frac{\delta y}{\left(\left|v_{y}^{\prime}\right|+C_{f y}\right)_{i, j, k}}, \frac{\delta z}{\left(\left|v_{z}^{\prime}\right|+C_{f z}\right)_{i, j, k}}\right)
$$

where $C_{\circ} \leq 1$ is the CFL number, and $C_{f x}, C_{f x}, C_{f x}$ are the fast magnetosonic waves speeds in the $x-, y-$, and $z$-directions respectively, and the minimum is taken over all grid cells. Since the background orbital flow becomes supersonic for $|x|>H / q$, where $H=C_{s} / \Omega_{0}$ is the scale height in the disk, the time step can be much larger using orbital advection in domains which span roughly $H$ or larger in the radial direction. This is the primary advantage of the method.

Since the first system of equations 49 through 52 ) are linear advection operators in one dimension, the numerical algorithms to integrate these equations are particularly simple. The finite volume discretization of the first three of these equations can be written as

$$
U_{i, j, k}^{n+1}=U_{i, j, k}^{n}-(\delta t / \delta y)\left(\bar{f}_{i, j+1 / 2, k}-\bar{f}_{i, j-1 / 2, k}\right)
$$

where $U$ represents each of the conserved quantities, and $\bar{f}_{i, j+1 / 2, k}$ the upwind fluxes of these quantities at cell interfaces in the $y$-direction. These fluxes are simply the integral of $U$ over the domain $v_{K} t$ upstream of the appropriate interface, for example

$$
\bar{f}_{i, j-1 / 2, k}=\int_{\left(y_{j-1 / 2}\right)-v_{k} \delta t}^{y_{j-1 / 2}} U^{n}\left(x_{i}, y, z_{k}\right) d y
$$

Numerically, this integral is converted into a finite sum over all the grid cells upstream of the interface in the $y$-direction. In general, the domain of dependence $v_{K} \delta t$ will span a non-integer number of cells. For those cells entirely contained in the domain, the volume-averaged value of $U$ is added to the sum. For the one cell which is only partially contained within the integral, then the higher-order reconstruction functions described in $\S 4$ of SGTHS are used to evaluate the fraction of the conserved variable that contributes to the integral. Note there is no time step constraint for stability of this algorithm. The domain of dependence can be arbitrarily large, and span any number of grid cells. The step simply represents a conservative remap of the solution in the $y$-direction by a distance $v_{K} \delta t$. 
There are two aspects to the orbital integration algorithm that warrant some discussion. The first is the integration of the induction equation. Note we have written equation 52 in a form that suggests the use of the CT algorithm with an effective emf given by $\mathcal{E}=-\mathbf{v}_{K} \times \mathbf{B}$. The components of this emf are

$$
\left(\mathcal{E}_{x}, \mathcal{E}_{y}, \mathcal{E}_{z}\right)=\left(-v_{K} B_{z}, 0, v_{K} B_{x}\right)
$$

The centering of the components of the emf used in Athena are shown in figure $1 \mathrm{in} \mathrm{SGTHS.} \mathrm{The} \mathrm{discrete} \mathrm{form} \mathrm{of} \mathrm{the} \mathrm{CT}$ update for each component of the magnetic field is given by equations 16 through 18 in SGTHS. The CT algorithm for orbital advection simply requires the calculation of the effective emf by integration of each component of the electric field $\mathcal{E}$ upstream of the appropriate cell edges distance $v_{K} \delta t$, that is

$$
\begin{gathered}
\mathcal{E}_{x, i, j-1 / 2, k-1 / 2}^{n+1 / 2}=-\int_{\left(y_{j-1 / 2}\right)-v_{K} \delta t}^{y_{j-1 / 2}} v_{K} B_{z}^{n}\left(x_{i}, y, z_{k-1 / 2}\right) d y \\
\mathcal{E}_{z, i-1 / 2, j-1 / 2, k}^{n+1 / 2}=\int_{\left(y_{j-1 / 2}\right)-v_{K} \delta t}^{y_{j-1 / 2}} v_{K} B_{x}^{n}\left(x_{i-1 / 2}, y, z_{k}\right) d y
\end{gathered}
$$

By using a CT discretization of equation 52 to evolve the magnetic field in the orbital advection step, we preserve the divergence-free constraint to machine round-off during the orbital advection step. Moreover, note that there are no source terms required in equation 52 The growth or decay of $B_{y}$ due to the shear is naturally captured in the CT difference formulae, avoiding any divergence-free interpolation as required in the algorithm of JGG.

The second aspect of the orbital advection step that requires further discussion is the integration of the energy equation 51. Note the source terms that appear on the RHS, which represent the work done by Reynolds and magnetic stress due to the radial shear of the orbital motion. The finite-volume discretization of equation 51 requires a time- and volume-averaged approximation for the source terms. In the Lagrangian frame (comoving with the fluid during the remap), all quantities except $B_{y}$ are constant, and the time evolution of $B_{y}$ in the Lagrangian frame is particularly simple, $B_{y}(t+\delta t)=B_{y}(t)+B_{x}\left(\partial v_{K} / \partial x\right) \delta t$, where $\partial v_{K} / \partial x=-q \Omega_{0}$ This suggest a "Lagrangian-step-plus-remap" algorithm for the energy equation. In the Lagrangian step, the total energy is updated using the time-averaged source terms

$$
\begin{aligned}
\hat{E}_{i, j, k}^{\prime} & =E_{i, j, k}^{\prime}-q \Omega_{0} \delta t B_{x, i, j, k}\left(B_{y, i, j, k}(0)-B_{x, i, j, k} q \Omega_{0} \delta t / 2\right) \\
& +q \Omega_{0} \delta t \rho v_{x, i, j, k}^{\prime} v_{y, i, j, k}^{\prime}
\end{aligned}
$$

where $B_{x, i, j, k}$ and $B_{y, i, j, k}(0)$ are the volume-averaged, cellcentered components of the magnetic field at the start of the orbital advection step. The remap step then uses equation 58 applied to the total energy with the source terms added, $\hat{E}_{i, j, k}^{\prime}$, to complete the update of the total energy. Comparisons of calculations of the MRI with an adiabatic equation of state, both with and without orbital advection (see next section), show that this algorithm for the energy source terms works well. Note that the only way in which the total volume averaged energy in the domain can change is via a non-zero total stress at the radial boundries. Additional tests have shown our method conserves the total energy if the stress at the boundaries is zero.

\subsection{Tests of Orbital Advection}

To test our orbital advection algorithm, we have run calculations in both two dimensions (in the $x-y$ plane), and full three dimensions. Our calculations span $-L_{x} / 2 \leq x \leq L_{x} / 2$, $-L_{y} / 2 \leq x \leq L_{y} / 2$, and $-L_{z} / 2 \leq z \leq L_{z} / 2$. We set the orbital frequency $\Omega_{0}=10^{-3}$, the shear parameter $q=3 / 2$, and unless otherwise stated adopt an isothermal equation of state with sound speed $C_{s}$ (we give the value of $C_{s}$ used for each test below). As before, we set the initial density $\rho_{0}=1$ and orbital velocity $v_{y, 0}=-q \Omega_{0} x$, and we use third-order reconstruction, the HLLC (for hydrodynamics) or HLLD (for MHD) Riemann solver, and the CTU+CT unsplit integrator for all the tests.

Our first test of orbital advection is the evolution of a hydrodynamic shearing wave (Johnson \& Gammie 2005, Balbus $\&$ Hawley 2006; Shen et al. 2006). We use a domain of size $L_{x}=L_{y}=1$, an isothermal equation of state with sound speed $C_{S}=1.29 \times 10^{-3}$, and an initial wavevector $2 \pi \mathbf{k}_{0} / L=(-8,2)$. We expect the maximum amplification of the wave amplitude to be 17 , at time $t \Omega_{0}=8 / 3$. Figure 5 compares the amplitude of the kinetic energy associated with the $x$-component of the velocity both with and without the orbital advection algorithm at various resolutions. In both cases, the evolution is accurately captured provided there are at least 8 grid points per wavelength in the initial conditions. Most importantly, there is virtually no aliasing in the solutions at late times. In both cases, the kinetic energy decays over 5 orders of magnitude, with no periods of sustained growth after the maximum.

Our first full MHD test of orbital advection is the advection of a weak $\left(\beta=2 \times 10^{6}\right)$ field loop. This test has been useful for developing the $\mathrm{CT}$ algorithm implemented in Athena (Gardiner $\&$ Stone 2005a; 2008; Stone et al. 2008). We use a domain of size $L_{x}=3$ and $L_{y}=8$, an isothermal equation of state with $C_{s}=10^{-3}$, and initialize the field loop following the method described in GS05 centered at the origin. To make the problem more complex, we introduce a uniform radial velocity $v_{x}=C_{s}$, which causes the loop to execute epicyclic oscillation as it is sheared. The amplitude of these oscillations is large enough so that the outer one quarter of the loop crosses the radial boundary at the extrema of the motion. This tests whether our implementation of the shearing box boundary conditions described in $\$ 4$ can maintain the integrity of the loop. Figure 6 shows plots of the field lines at four times in the evolution. Note there are no indications of the stripes which appear if the EMFs are computed incorrectly (see figures 2 and 3 in GS05), confirming the upwind CT method developed by GS05 to compute the emfs also works well with orbital advection. In addition, there are no features in the loop associated with the boundaries, indicating our implementation of the shearing box boundary conditions is accurate.

Another sensitive MHD test recently introduced by JGG is the evolution of a compressible shearing wave (Johnson 2007). We have repeated the test shown in figure 11 of JGG, and compared the resulting solution to a numerical integration of the ODEs that describe the analytic solution to the problem kindly provided to us by B. Johnson. The calculation uses a domain 
of size $L_{x}=L_{y}=L_{z}=L=0.5$, and a numerical resolution of $N \times N / 2 \times N / 2$, where $N=16,32$, and 64 (these values are chosen to match those used by JGG). An isothermal equation of state with $C_{s}=1$ is adopted, and for this test we use $\Omega_{0}=1$. Although the details of how to initialize such tests are given in the Addendum to JGG, to be specific we give the initial conditions we actually used in Athena for this test, namely density $\rho=1+5.48082 \times 10^{-6} \cos \left(\mathbf{k}_{0} \cdot \mathbf{x}\right)$, velocity fluctuations $\mathbf{v}^{\prime}=$ $\left(-4.5856 \times 10^{-6}, 2.29279 \times 10^{-6}, 2.29279 \times 10^{-6}\right) \cos \left(\mathbf{k}_{0} \cdot \mathbf{x}\right)$, and magnetic field $\mathbf{B} / \sqrt{4 \pi}=\left(5.48082 \times 10^{-7}, 1.0962 \times\right.$ $\left.10^{-6}, 0\right) \cos \left(\mathbf{k}_{0} \cdot \mathbf{x}\right)+(0.1,0.2,0)$, where the wavevector $\mathbf{k}_{0}$ has components $(-2,1,1)(2 \pi / L)$. Figure 7 shows the time evolution of the volume-averaged perturbations in the real part of the azimuthal component of the magnetic field, defined as

$$
\delta B_{y}=\int_{V} 2\left(B_{y}-\hat{B}_{y}\right) \cos (\mathbf{k}(t) \cdot \mathbf{x})
$$

where $B_{y}$ is the numerical solution computed by Athena, $\hat{B}_{y}=$ $0.2-0.15 \Omega_{0} t$ is the expected evolution of the real part of the field, and the wavevector has components $\mathbf{k}(t)=(-2+$ $\left.q \Omega_{0} t, 1,1\right)(2 \pi / L)$. Note the highest resolution solution, which has 32 grid points per wavelength in each direction initially, is indistinguishable from the semi-analytic solution.

It is instructive to compare the nonlinear saturation of the MRI in calculations both with and without orbital advection. We present the evolution of two different threedimensional calculations, one with orbital advection and the other without. Both start with no net vertical flux, $\mathbf{B}=$ $\left(0,0,\left(2 p_{0} / \beta\right)^{1 / 2} \sin 2 \pi x / L_{x}\right)$, where $\beta=400$, use a domain of size $L_{x}=L_{y}=8 H$, and $L_{z}=H$ (where $H=C_{s} / \Omega_{0}=1$ ), and adopt an isothermal equation of state with $C_{s}=10^{-3}$. The resolution is $32 / H$ in each dimension. Figure 8 plots the time evolution of the magnetic energy in both cases. In the nonlinear regime, the timestep in the calculation with orbital advection was on average 4.3 times larger than the calculation without. The results show the solutions are in excellent qualitative agreement. Because the nonlinear evolution of the MRI is chaotic (Winters et al. 2003), we do not expect exact agreement, but only the same values for suitably time-averaged quantities. The level of agreement between our two calculations is similar to that reported by JGG. These authors point out that because the truncation error varies with $x$ in the shearing box without orbital advection, there are unphysical patterns in the radial profile of time-averaged quantities such as the stress and density. Figure 9 plots these profiles for this calculation. Note that without orbital advection, we observe a minimum in the density, and a maximum in the stress, near the center of the calculation. This is likely caused by a minimum in the truncation error in the region of this point, since the velocity amplitude (including the radial, azimuthal, and vertical components) is a minimum near there. However, with orbital advection the profiles of the density and stress are clearly much smoother; they only show small amplitude variations associated with the MRI-driven turbulence.

These calculations do not use the corrections to the shearing box boundary conditions (Gressel \& Ziegler 2007) that are required to conserve mass and momentum exactly (energy is not conserved with an isothermal equation of state). Nonetheless, we find that at the end of the calculation without orbital advection, mass is conserved to one part in $10^{3}$, and with orbital advection it is conserved to one part in $10^{5}$. Since these simulations are only run for 16 orbits, we have also checked mass conservation at various resolutions for zero net vertical simulations in a domain of size $L_{x}=H, L_{y}=4 H$, and $L_{z}=H$, using resolutions of $32 / H, 64 / H, 128 / H$, and $256 / H$. This study was conducted to confirm the lack of convergence of the amplitude of the Maxwell stress in zero-net flux unstratified boxes discussed by Fromang et al. (2007), and indeed we confirm this result. A variety of other results from this study will be reported elsewhere. Here we note that without orbital advection, mass conservation can be a problem at the lowest resolutions. At $32 / H$, roughly $8 \%$ of the initial mass is lost by 100 orbits, with the mass loss rate essentially constant with time. The mass loss rate decreases with resolution, however even at $256 / H, 1.5 \%$ of the mass is lost by 100 orbits. The use of orbital advection greatly improves conservation. After 100 orbits, we find only $0.03 \%$ of the initial mass is lost by 100 orbits with orbital advection, and this number is independent of resolution.

Finally, we have also used simulations of the MRI with an adiabatic equation of state to investigate the implementation of the source terms in the energy equation in the orbital advection step. We have repeated a simulations with net vertical flux $\mathbf{B}=\left(0,0,\left(2 p_{0} / \beta\right)^{1 / 2}\right)$, where $\beta=400$, in a domain of size $L_{x}=H, L_{y}=2 \pi H$, and $L_{z}=H$, using a resolution of $64 \times 128 \times 64$. This is a repeat of run Z2 in Gardiner \& Stone (2005b). The calculation starts with $p_{0}=10^{-6}$, and uses $\gamma=5 / 3$. We have run calculations both with and without orbital advection, and compared the resulting time evolution of the total energy with figure 3 in Gardiner \& Stone (2005b). Again, because of the chaotic nature of the MRI, we do not recover exact agreement in the two calculations, however both show growth of the total energy at very similar time-averaged rates, with all of the increase after saturation occurring in the internal energy density.

\section{APPLICATION TO THE MRI}

The test results presented in the previous section demonstrate the orbital advection algorithm is at least as accurate (although perhaps not any more accurate) than integrations that do not use it. The primary advantage of orbital advection, however, is not that it is more accurate, but that it removes the background shear flow from the time step stability limit, and therefore enables much more efficient studies of accretion flows over a wide range of radii. Moreover, the new method based on CT introduced in this paper is simpler than previous approaches, and preserves the divergence free constraint to machine precision.

Figure 10 shows images of the density and azimuthal velocity fluctuations in a shearing box simulation of the MRI in a very wide domain of size $L_{x}=L_{y}=32 H$, and $L_{z}=H$, using a resolution of $32 / H$ in each dimension, or $1024 \times 1024 \times 32$. The initial conditions and parameters of the calculation are identical to that presented in figure 8. Interestingly the spiral density waves excited by the MRI are immediately obvious, and have the same 
pitch angle as in smaller domains, indicating the box size does not determine these features (Heinemann \& Papaloizou 2008a; b). Recently Johansen et al. (2008) have reported long-lived density features in domains with wide vertical extent, and a more careful analysis of this and other runs reproduces this result. The time averaged Maxwell stress in this calculation is little different than the value in much smaller domains, indicating the turbulent stress must be localized on scales $\lesssim H$ (Guan et al. 2009).

JGG found unphysical features in the time-averaged radial density profile even with orbital advection, wherever the orbital shear displacement was close to an integer number of zones. In figure 11 we plot this profile for the calculation shown in figure 10. Using the time step measured from this simulation, the radial locations where $\delta t q \Omega_{0} / \delta y$ is an integer are plotted as vertical dashed lines. There are no significant extrema at these locations, indicating the stress and truncation error are smooth with radius. This is another advantage of the MHD orbital advection algorithm developed in this paper.

In summary, we have described the inclusion of source terms for the shearing box approximation in the Athena MHD code, including a Crank-Nicholson time differencing that preserves the amplitude of epicyclic oscillations exactly. We have also described an orbital advection algorithm based on CT for evolving the induction equation to preserve the divergence free constraint on the magnetic field. We have shown this algorithm provides more accurate solutions at less computational cost. These algorithms have already been used to study hydrodynamic turbulence in the shearing box (Shen et al. 2006). A number of new studies of the MRI in wide radial domains in both unstratified, and vertically stratified disks are underway (Davis et al. 2010).

We thank Sebastien Fromang, Charles Gammie, John Hawley, Bryan Johnson, E. Ostriker, and Jake Simon for helpful discussions, and S. Fromang for providing the initial conditions for the nonlinear density wave test, and B. Johnson for providing the reference solution for the MHD shearing wave test. Financial support was provided by the DOE through DEFG52-06NA26217. Simulations were performed on computational facilities at the Princeton Institute for Computational Science and Engineering, and through resources provided by NSF grant AST-0722479.

\section{REFERENCES}

Balbus, S.A., \& Hawley, J.F., 1991. ApJ, 376, 214.

Balbus, S.A., \& Hawley, J.F., 2003. LNP, 614, 329.

Balbus, S.A. 2003. ARA\&A, 41, 555

Balbus, S.A., \& Hawley, J.F., 2006. ApJ 652, 1020.

Bodo, G., Mignone, A., Cattaneo, F., Rossi, P., \& Ferrari, A. 2008. A\&A 487, 1 .

Davis, S.W., Stone, J.M., \& Pessah, M., 2010. ApJ 713, 52.

Evans, C.R., \& Hawley, J.F., 1988. Ap.J., 322, 659

Fromang, S., \& Papaloizou,J., 2007. A\& A, 468, 1.

Fromang, S., Papaloizou, J., Lesur, G., \& Heinemann, T. 2007. A\&A 476, 1123

Gardiner, T.A., \& Stone, J.M., 2005a. JCoPh, 205, 509

Gardiner, T.A., \& Stone, J.M., 2005b. In Magnetic Fields in the Universe, AIP Conf. Proc. 784, 475

Gardiner, T.A., \& Stone, J.M., 2008. JCoPh, 227, 4123

Gressel, O., \& Ziegler, U. 2007. CoPhC, 176, 652.

Gressel, O., 2010. MNRAS, in press.

Guan, X., Gammie, C.F., Simon, J.B., \& Johnson, B.M. 2009. ApJ 694, 1010.

Hawley, J.F., \& Balbus, S.A., 1992. ApJ 400, 595.

Hawley, J.F., Gammie, C.F., \& Balbus, S.A., 1995. ApJ 440, 742

Heinemann, T., \& Papaloizou, J.C.B., 2008a. arXiv0812.2068.

Heinemann, T., \& Papaloizou, J.C.B., 2008b. arXiv0812.2471.

Hill, G.W., 1878. Amer. J. Math. 1, 5.

Hirose, S., Krolik, J., \& Blaes, O., 2009. ApJ 691, 16.

Ilgner, M., \& Nelson, R.P. 2008. A\&A, 483, 815.
Johansen, A., Youdin, A., \& Klahr, H. 2009. ApJ 697, 1269.

Johnson, B.M., \& Gammie, C.F. 2005. ApJ 626, 978.

Johnson, B.M. 2007. ApJ 660, 1375.

Johnson, B.M., Guan, X., \& Gammie, C.F. 2008a. ApJS 177, 373. Addendum: ApJS 179, 553.

Lesur, G., \& Longaretti, P.-Y., 2007. A\&A, 378, 1471.

Masset, F., 2000. A\& AS, 141, 165.

Piontek, R.A., Gressel, O., \& Ziegler, U. 2009. A\& A 499, 633.

Regev, O., \& Umurhan, O.M., 2008. A\& A, 481, 21

Shen, Y., Stone, J.M., \& Gardiner, T.A., 2006. ApJ 653, 513

Simon, J.B., \& Hawley, J.F., 2009. ApJ 707, 833.

Simon, J.B., Hawley, J.F., \& Beckwith, K., 2009. ApJ 690, 974.

Stone, J.M., Hawley, J.F., Gammie, C.F., \& Balbus, S.A. 1996. ApJ 463, 656.

Stone, J.M. \& Gardiner, T.A., 2005. In Magnetic Fields in the Universe, AIP Conf. Proc. 784, 16

Stone, J.M., Gardiner, T.A., Teuben, P., Hawley, J.F., \& Simon, J.B., 2008 ApJS 178, 137 (SGTHS)

Stone, J.M., \& Gardiner, T.A., 2009. NewA 14, 139.

Stone, J.M. 2009. ASP Conf. Proc. 406, 277.

Tilley, D.A., Balsara, D.S., Brittain, S.D., \& Rettig, T., 2010. MNRAS 403, 211.

Turner, N.J., \& Sano, T., 2008. ApJ 679, L131.

Winters, W.F., Balbus, S.A., \& Hawley, J.F. 2003. MNRAS 340, 519. 


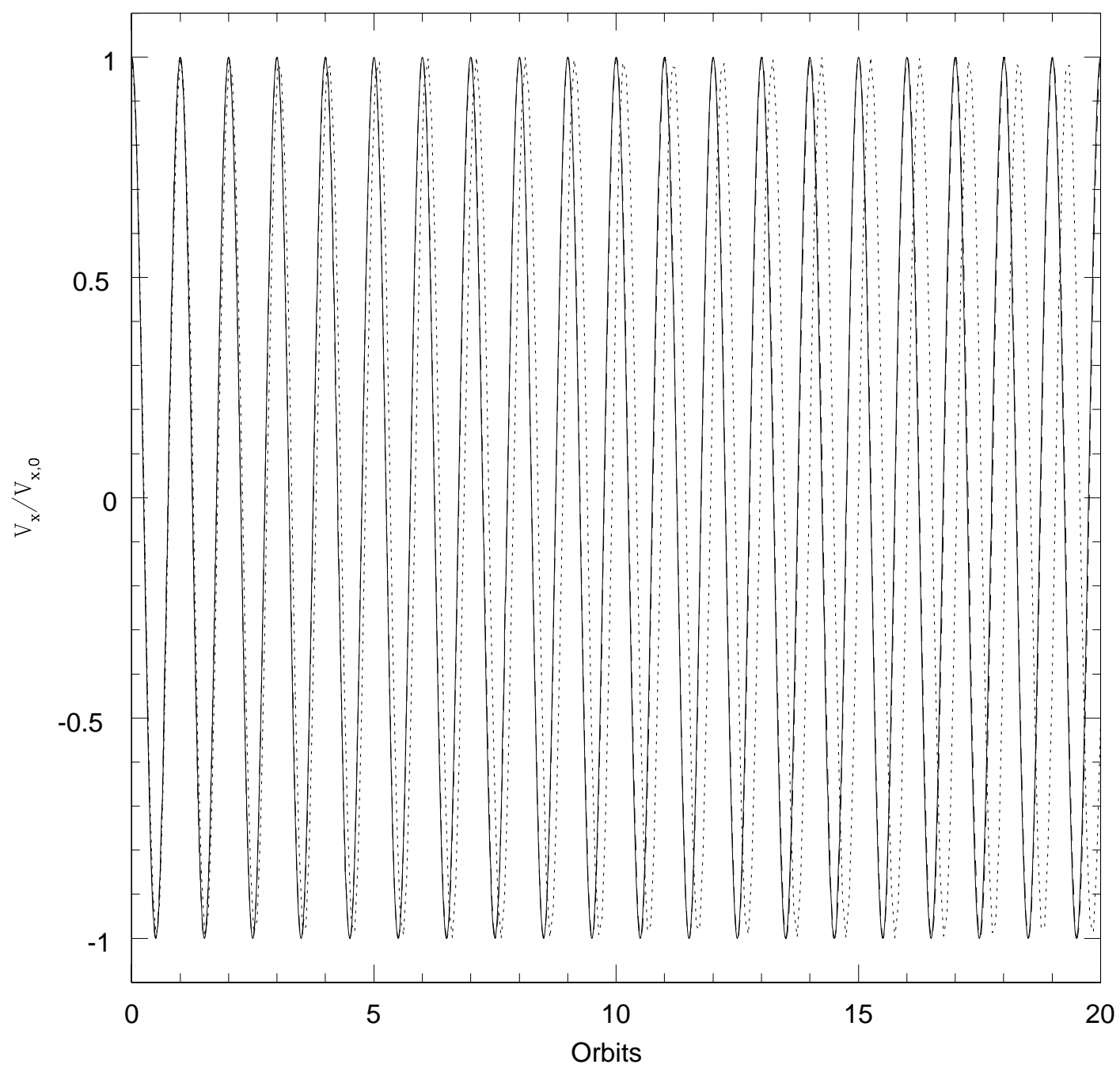

FIG. 1. - Time evolution of radial velocity amplitude, normalized by the initial value, in epicyclic motion in a box of radial extent $L_{x}=1$ (solid line), $L_{x}=10$ (dashed line), and $L_{x}=50$ (dotted line) computed on a grid of $64^{2}$. There is never amplitude error in the solutions, and only small phase error in the largest box (in which the timestep is about 0.1 orbits).
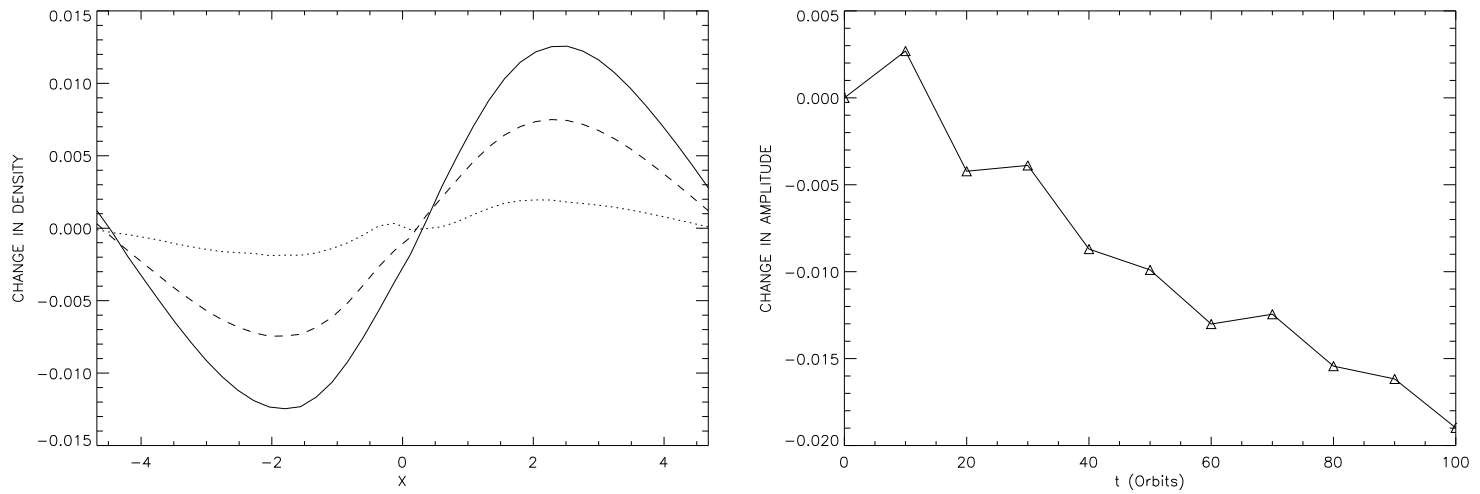

FIG. 2.- (Left). Fractional change in density in an axisymmetric nonlinear density wave at $t=10 T$ (dotted line), $50 T$ (dashed line), and $100 T$ (solid line), where $T=5254.31$ is the wave period, on a grid of 40 cells. (Right). Fractional change in the wave amplitude as a function of time. Both quantities are defined in the text. 

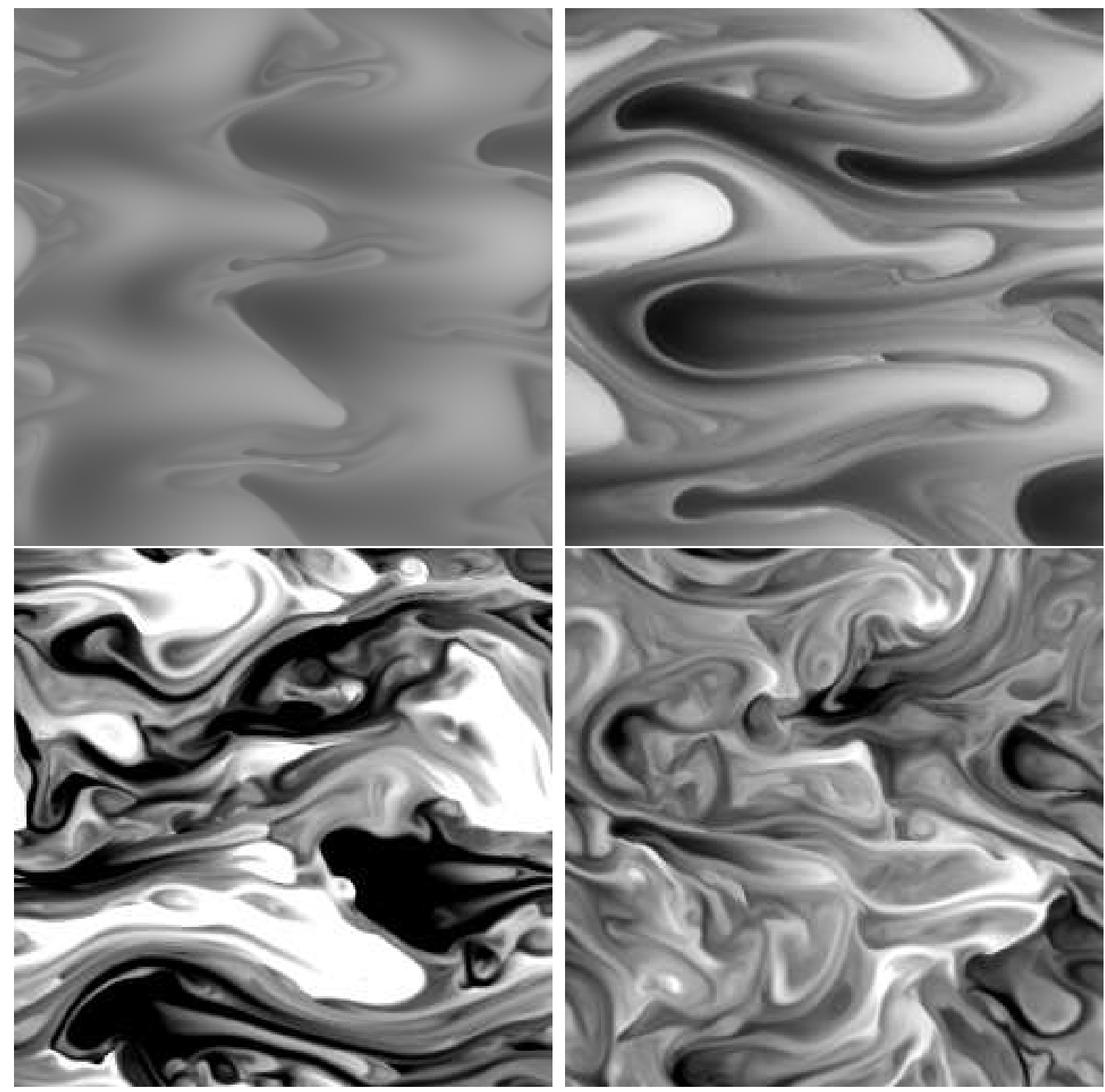

FIG. 3.- Images of the azimuthal velocity fluctuations $\delta v_{y}=v_{y}-0.75 \Omega_{0} x$ in the axisymmetric MRI computed with a grid of $256^{2}$. From left-to-right and top-to-bottom the images are shown at times of $3.45,3.70,4.0$, and 6.0 orbits respectively. The images are scaled over $\pm 6 \times 10^{-4}$. 


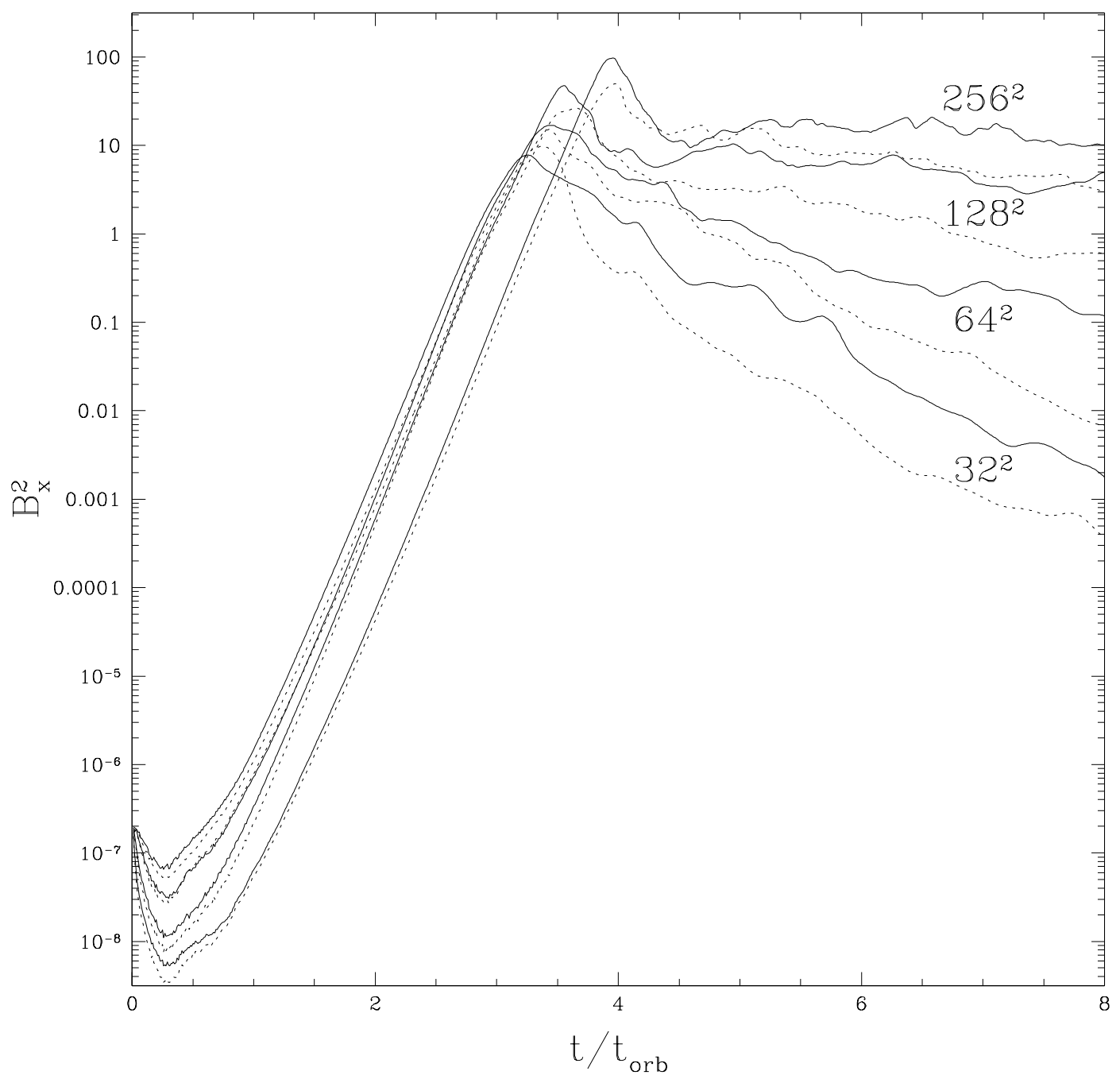

FIG. 4.- Time evolution of $0.5 B_{x}^{2}$ in the axisymmetric MRI. The solid lines are for third-order spatial interpolation, the dashed lines are for second-order. The numerical resolution of each pair of lines is labeled. 

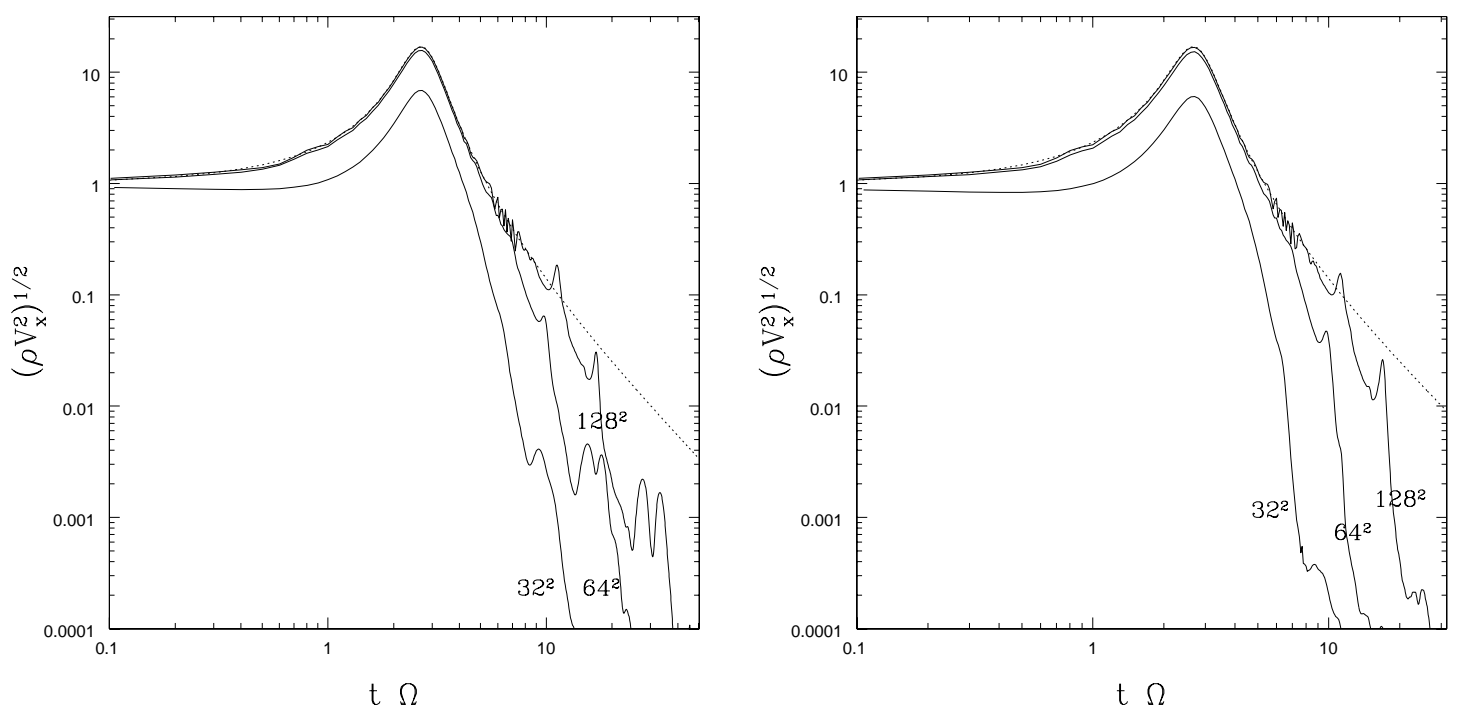

FIG. 5.- Time evolution of the radial velocity amplitude in an incompressible shearing wave at numerical resolutions of $32^{3}, 64^{3}$, and $128^{2}$, corresponding to 4 , 8 , and 16 grid points per wavelength initially. Solutions computed both without orbital advection (left), and with orbital advection (right) are shown. The dashed line in both cases is the analytic solution, and for each curve the amplitude is normalized by the initial value. Particularly notable is the extremely small level of aliasing.
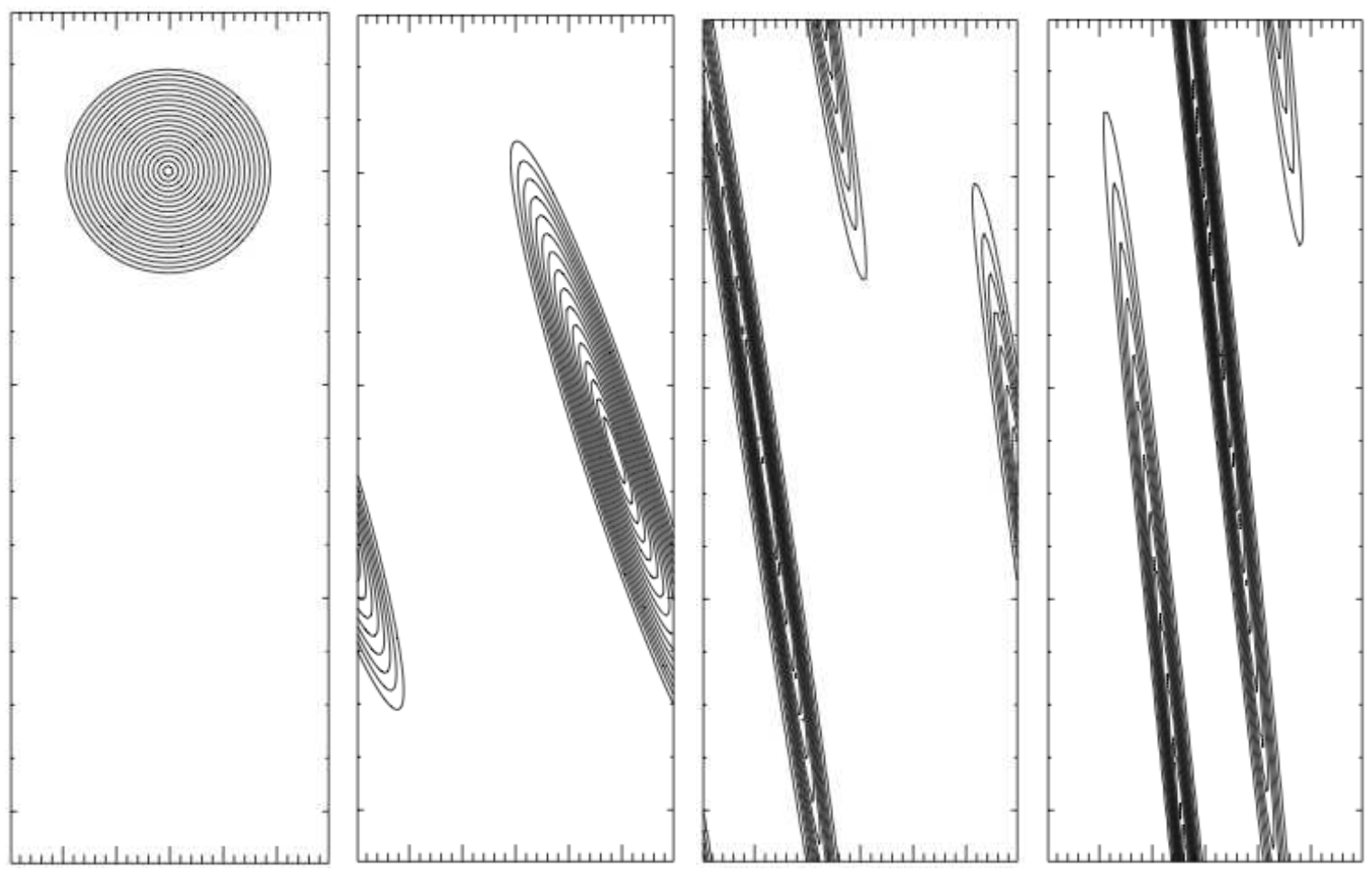

FIG. 6.- Field lines of an initially circular field loop advected by epicyclic motion and sheared by the background flow, computed using orbital advection. Twenty field lines are shown at each times of (from left to right) $t=0,0.3,0.7$, and 1 orbit. 


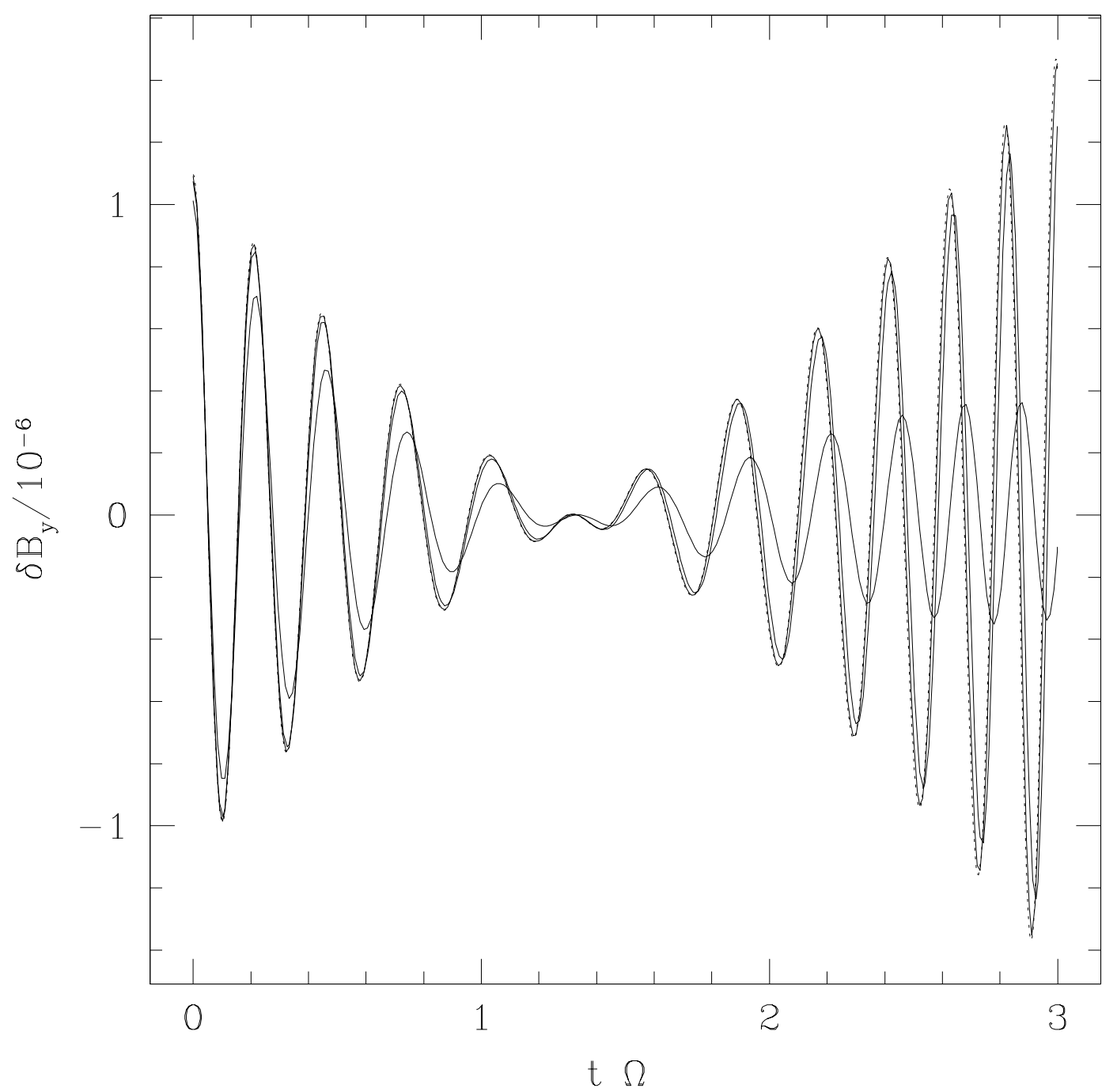

FIG. 7.- Time evolution of the real part of the wave amplitude in the azimuthal component of the magnetic field for an MHD shearing wave test, computed using orbital advection. The solid lines are at a resolution of 8,16 , and 32 grid points per $L_{z}$, while the dashed line is the semi-analytic solution. 


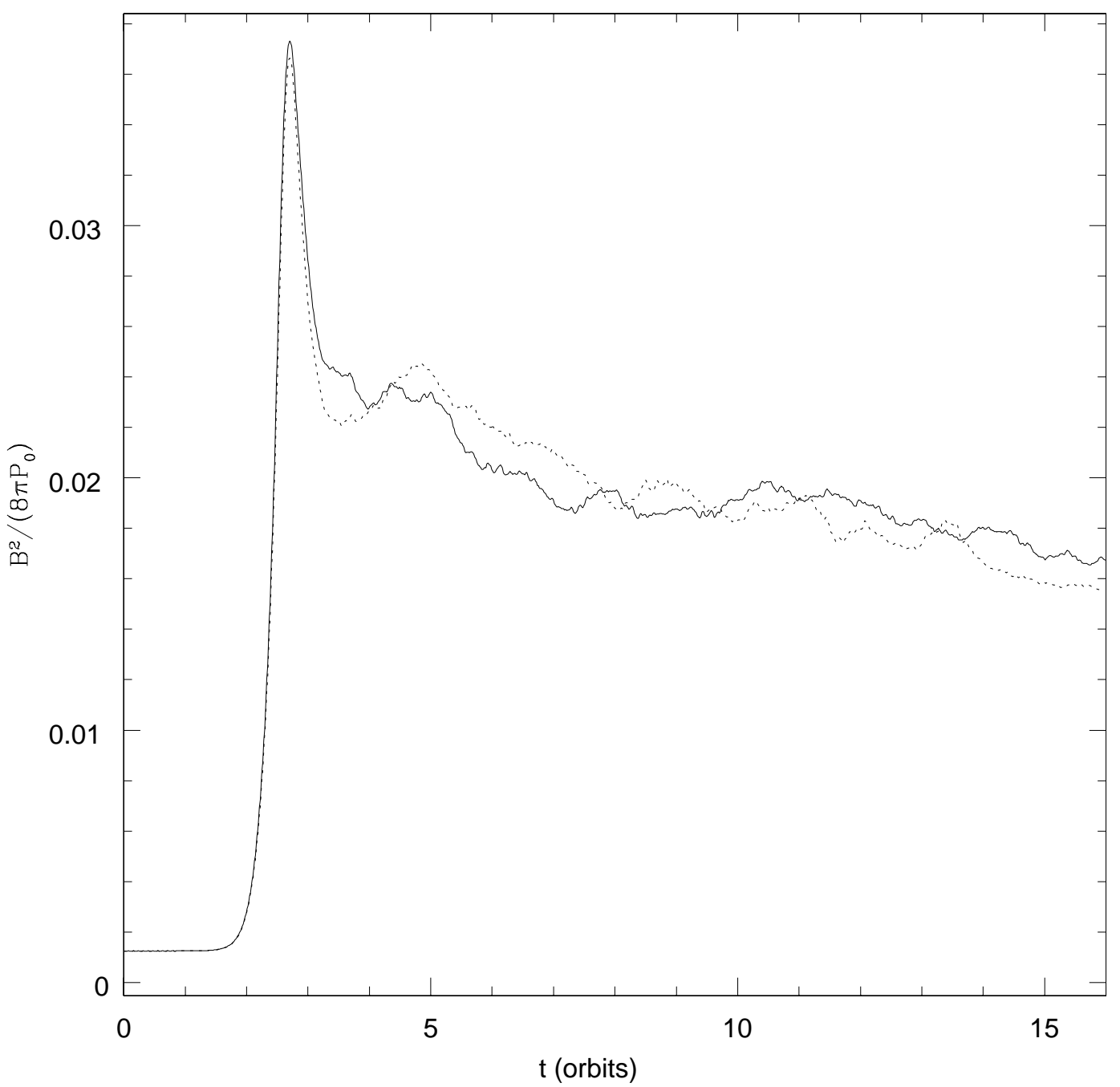

FIG. 8. - Time evolution of the magnetic energy in a zero-net flux MRI calculation in a box with radial dimension $L_{x}=8 H$. The solid line is computed with orbital advection, the dashed without.
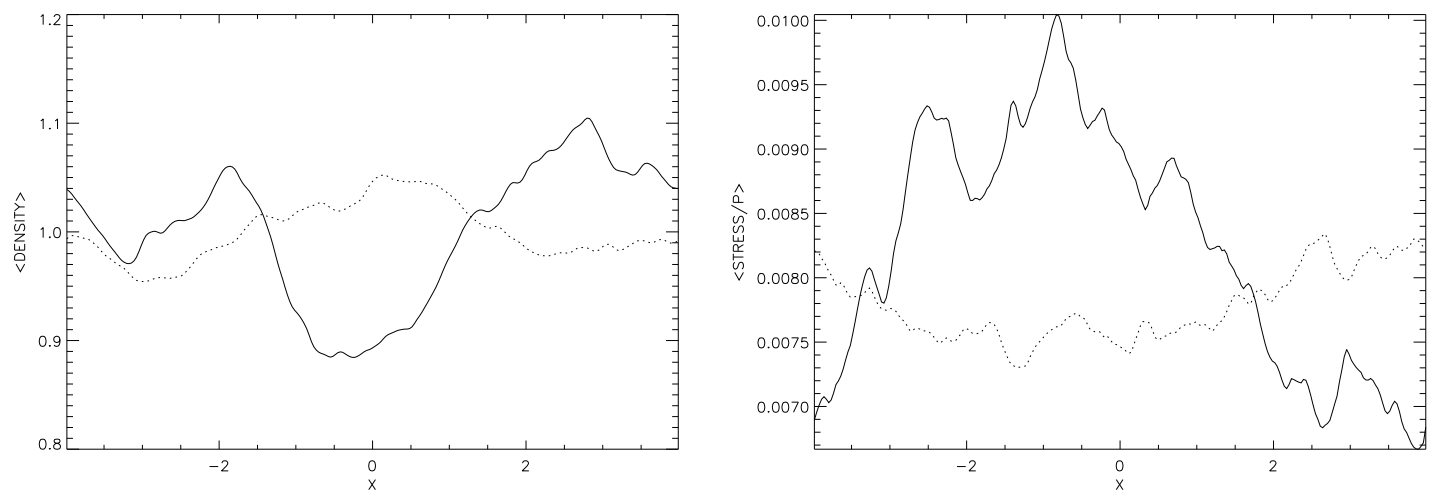

FIG. 9.- Radial profile of the average density (left) and stress (right) in a zero-net flux MRI calculation computed with (dashed line) and without (solid line) orbital advection. The averages are taken over all $y$ and $z$, and over orbits 7-16 for the solid line, and 70-100 for the dashed line. 

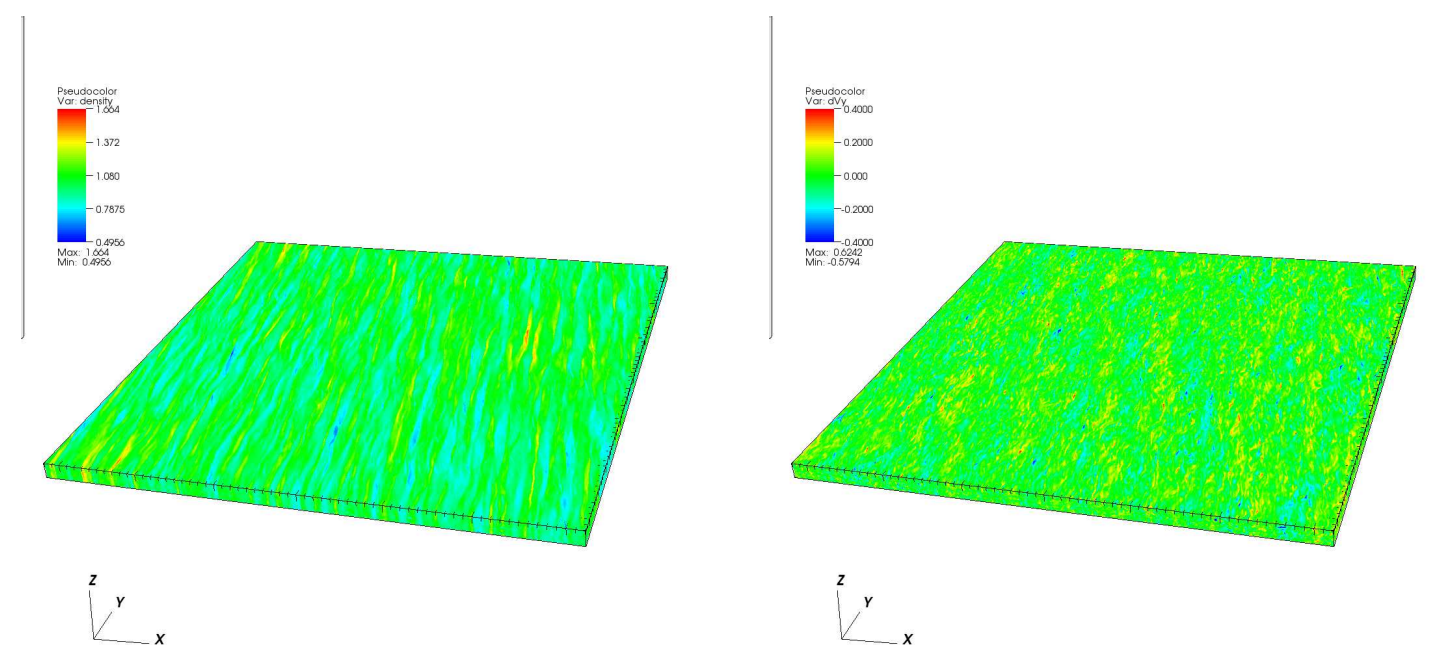

FIG. 10.- Images of the density (left) and azimuthal velocity fluctuations scaled to the sound speed (right) from a zero-net flux MRI calculation in a box with radial dimension $L_{x}=32 \mathrm{H}$ at $t=16$ orbits, computed with orbital advection.

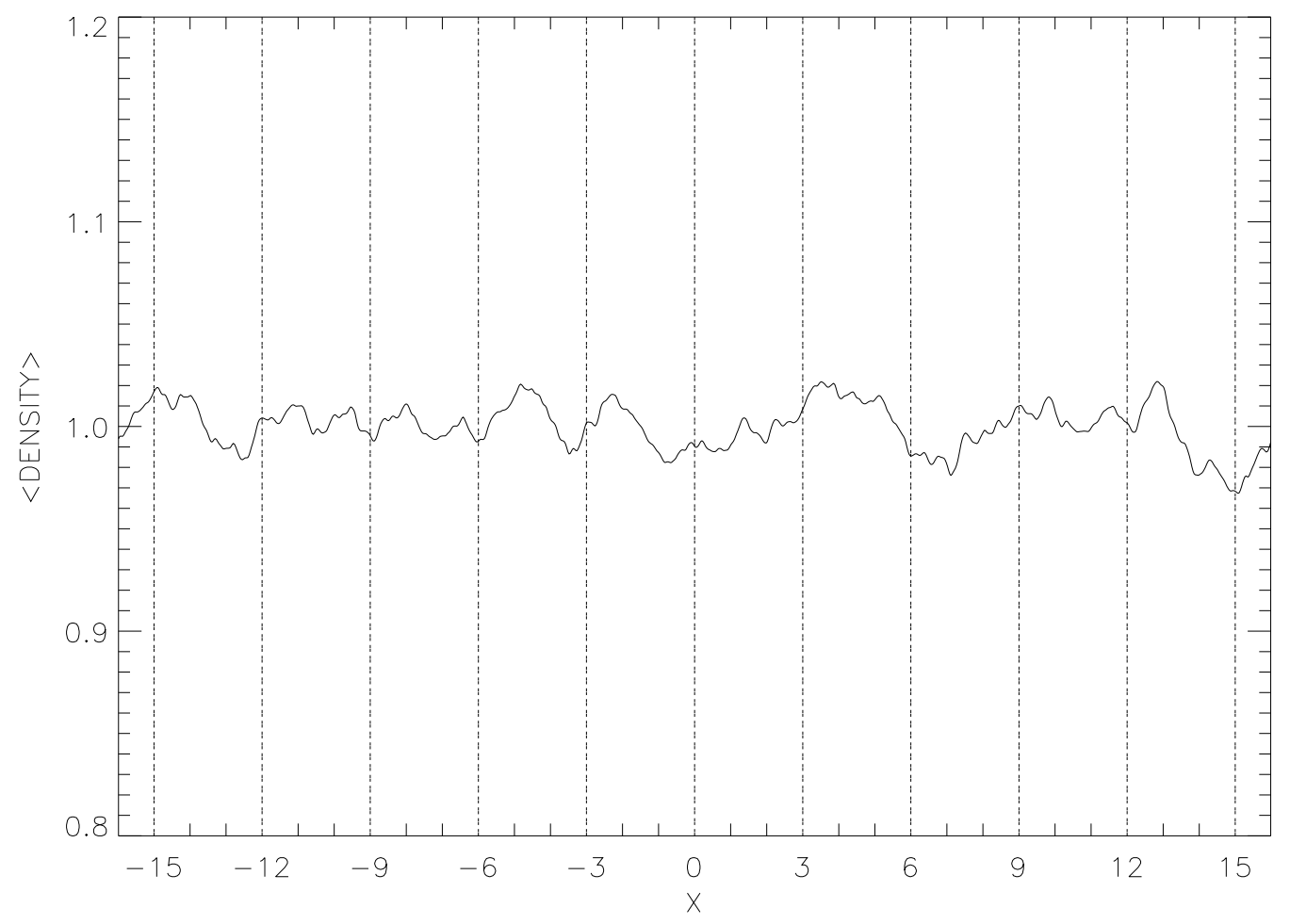

FIG. 11.- Radial profile of the average density in a zero-net flux MRI calculation in a box with radial dimension $L_{x}=32 H$. The average is taken over all $y$ and $z$, and over orbits 7-16. For the typical timestep used in the calculation the azimuthal displacement in the orbital advection algorithm is an integer number of zones at the locations marked by the vertical dashed lines. There is no evidence of density minima at these locations. 